\title{
Vectorial Phase Retrieval of 1-D Signals
}

\author{
Oren Raz, Nirit Dudovich, and Boaz Nadler, Member, IEEE
}

\begin{abstract}
Reconstruction of signals from measurements of their spectral intensities, also known as the phase retrieval problem, is of fundamental importance in many scientific fields. In this paper we present a novel framework, denoted as vectorial phase retrieval, for reconstruction of pairs of signals from spectral intensity measurements of the two signals and of their interference. We show that this new framework can alleviate some of the theoretical and computational challenges associated with classical phase retrieval from a single signal. First, we prove that for compactly supported signals, in the absence of measurement noise, this new setup admits a unique solution. Next, we present a statistical analysis of vectorial phase retrieval and derive a computationally efficient algorithm to solve it. Finally, we illustrate via simulations, that our algorithm can accurately reconstruct signals even at considerable noise levels.
\end{abstract}

Index Terms-Convex relaxation, 1-D phase retrieval, signal recovery from modulus Fourier measurements, statistical model selection.

\section{INTRODUCTION AND MAIN RESULTS}

$\mathbf{I}$ $\mathrm{N}$ a variety of experimental setups, while the signal of interest, either in the time or spatial domain, cannot be directly measured, it is often possible to measure the absolute value of its Fourier transform. Unfortunately, in such measurements, the phase information, crucial for complete reconstruction of the signal, is lost. The challenge is to estimate this phase, typically under some assumptions on the signal of interest.

This inverse problem, known as phase retrieval, is of fundamental importance in a broad range of scientific fields [1], [2]. Phase retrieval applications range from X-ray crystallography [3], nuclear magnetic resonance [4], astrophysics [5], lens-less imaging [6]-[11] and radar [12] to characterization of ultrashort pulses [13], [14].

Mathematically, phase retrieval poses several theoretical and computational challenges, including questions of uniqueness, development of efficient reconstruction algorithms and analysis of their convergence properties. Despite decades of research, phase retrieval is still a challenging and not yet fully resolved problem. For example, in 1-D the phase retrieval problem is in general ill posed, admitting multiple solutions [15]. In contrast,

Manuscript received June 18, 2012; revised October 31, 2012 and November 28, 2012; accepted December 13, 2012. Date of publication January 14, 2013; date of current version March 08, 2013. The associate editor coordinating the review of this manuscript and approving it for publication was Prof. Emmanuel Candes. This work was supported in part by grants from the Israeli Science Foundation (ISF), the Minerva foundation, and the Crown center for photonics.

O. Raz and N. Dudovich are with the Physics Faculty, Weizmann Institute of Science, Rehovot, 76100 Israel (e-mail: oren.raz@weizmann.ac.il; nirit.dudovich@weizmann.ac.il).

B. Nadler is with the Department of Computer Science and Applied Mathematics, Weizmann Institute of Science, Rehovot, 76100 Israel (e-mail: boaz. nadler@weizmann.ac.il).

Color versions of one or more of the figures in this paper are available online at http://ieeexplore.ieee.org.

Digital Object Identifier 10.1109/TSP.2013.2239994 for 2-D signals with noise-free measurements, under various assumptions phase retrieval has a unique solution [15], though computing it in practice is a non-trivial task. The two main approaches, pioneered by Gerchberg and Saxton [16] and Fienup [17] are direct solutions and iterative search. The former are limited by the size of the images, whereas the latter by efficiency and convergence [18]. Moreover, due to the non-convexity of the classical phase retrieval problem, relatively little is understood about the convergence or robustness to measurement noise of current algorithms [2], [19]. For a review of phase retrieval, we refer the reader to [1], [18], [20], [21] and references therein.

1) Main Results: In this paper we present a novel framework for the phase retrieval problem that overcomes some of these challenges. Based upon our preliminary work [22], the key idea underlying our approach is that in many physical systems, one can measure not only one, but rather several signals, and their pairwise interferences. In the simplest setting, this involves measuring, at a finite set of frequencies, the absolute Fourier coefficients of two signals $f_{1}(t)$ and $f_{2}(t)$ and of their interferences $f_{1}(t)+f_{2}(t), f_{1}(t)+\mathbf{i} f_{2}(t)$, namely $\left|F_{1}(w)\right|,\left|F_{2}(\omega)\right|$, $\left|F_{1}(\omega)+F_{2}(\omega)\right|$ and $\left|F_{1}(\omega)+\mathbf{i} F_{2}(\omega)\right|$. In this new framework, denoted vectorial phase retrieval (VPR), the problem is to retrieve the phases of the two signals from these four spectral measurements.

Experimentally, vectorial phase retrieval can be implemented in various physical systems. One example is when the signal of interest has a vectorial nature, such as light polarization [22] or spin of matter waves. Another example is spatial-temporal coupling of signals as in lateral shearing interferometry [23]. While VPR can be realized also for 2-D images, in this paper we focus for simplicity on the 1-D case. From a mathematical viewpoint, vectorial phase retrieval raises two fundamental questions, which are the focus of this paper: i) uniqueness of the solution; and ii) computationally efficient and robust to noise vectorial phase retrieval algorithm .

Our main contributions are as follows:

1) Formulation and Uniqueness of Vectorial Phase Retrieval: We formulate this novel framework and study its uniqueness in the noise-free case. Specifically, we show that in the absence of noise, VPR admits a unique solution even in the simplest setting of only two independent 1-D signals with finite support.

2) Statistical noise model and analysis: We present a principled approach to handle measurement noise. We propose to retrieve the unknown phases by minimizing a carefully constructed quadratic functional, and a simple criterion to estimate the unknown support of the signals.

3) Direct solution: We further relax the non-convex phase constraints to a convex optimization problem, requiring the solution of a set of linear equations. This yields a novel phase retrieval algorithm which is both computationally efficient and empirically robust to noise. 
The key point of our work is that measuring several signals and their interferences alleviates some of the computational challenges associated with classical phase retrieval. Even in the presence of noise, the unknown phase can be estimated by solving a set of linear equations. As such, and given the feasibility of spectral interference measurements in a broad range of applications, vectorial phase retrieval presents a promising new approach to this long-standing problem.

2) Related Works: Several works considered modified phase retrieval problems, based on additional measurements. For example, [24], [25] suggested to overcome the ambiguities of 1-D phase retrieval with additional exponential apodization measurements. Another phase retrieval method based on multiple measurements with known relations between them is the transport of intensity equation [26], [27].

In holography [21] the phase is extracted from measurements of both $|F(\omega)|$ and $|F(\omega)+H(\omega)|$, with $H(\omega)$ a known reference signal. Recently, for 2-D holography, [28] showed that reconstruction is possible even if $H(\omega)$ contains a few severe errors or has few missing values. The main differences between holography and our method are that we do not assume a known $H(\omega)$ but rather only that it corresponds to a signal $h(t)$ with a finite support, and additionally we measure $|H(\omega)|$.

A different approach is phase retrieval from structured illuminations of the same object with several optical masks [29], [30]. The problem is to recover the signal $f$ from modulus measurements of many dot products $\left|\left\langle f, \mathbf{z}_{i}\right\rangle\right|$. As shown in [29], [31], this problem can be solved by convex programming via a low rank matrix completion formulation. As proven in [32], with high probability, this formulation is robust to measurement noise, and in fact (w.h.p.) yields the unique solution in the noise-free case, see also [33]. A different semi-definite program approach to recover the phases from noise-free measurements was recently derived in [34]. Lastly, [35] also studied recovery of $f$ from measurements of $\left|\left\langle f, \mathbf{z}_{i}\right\rangle\right|$ when $\mathbf{z}_{i}$ form a frame in a Hilbert space, and derived fast algorithms to do so.

While all of these works are related to our approach, both our measurement setup and method of solution are nonetheless quite different. The rest of the paper is organized as follows. In Section II we present our problem setup. Uniqueness of VPR in the absence of noise is proven in Section III, whereas the effect of noise is studied in Section IV. In Section V we present a novel algorithm for vectorial phase retrieval. Its empirical performance is evaluated in Section VI. We conclude with a summary and discussion in Section VII.

\section{Problem Setup}

Notations: We denote by $\mathbf{i}=\sqrt{-1}$. For $\mathbf{z} \in \mathbb{C}$, we denote its complex conjugate by $\bar{z}$ and by $\Re(z), \Im(z)$ its real and imaginary parts, respectively. For $\mathbf{x} \in \mathbb{C}^{n}, \mathbf{x}^{H}$ denotes its Hermitian transpose, whereas $\|\mathbf{x}\|$ denotes its Euclidean norm, $\|\mathbf{x}\|^{2}=\sum_{j}\left|x_{j}\right|^{2}$. The normal distribution with mean $\mu$ and variance $\sigma^{2}$ is denoted $\mathcal{N}\left(\mu, \sigma^{2}\right)$, whereas the circularly symmetric complex normal distribution is $\mathcal{C N}\left(\mu, \sigma^{2}\right)$. The expectation of a random variable $X$ is denoted by $\mathbb{E}[X]$.

1) Spectral Measurements: While phase retrieval problems apply also to spatial signals, in what follows we consider for simplicity only temporal signals. Let $f_{c}(t)$ be a continuous 1-D signal and let $F(\omega)$ be its Fourier transform, decomposed into its phase and absolute magnitude,

$$
F(\omega)=\int f_{c}(t) e^{-\mathbf{i} \omega t} d t=|F(\omega)| e^{\mathbf{i} \phi(\omega)} .
$$

We assume $f_{c}(t) \in L_{1}(\mathbb{R}) \cap L_{2}(\mathbb{R})$ so (1) is well defined.

As mentioned in the introduction, in many applications direct measurement of $f_{c}(t)$ is not feasible. Yet, it is possible to sample $|F(\omega)|^{2}$, at $n$ typically equispaced frequencies $\omega_{j}=j \Delta \omega$, where $\Delta \omega$ is the spectral resolution of the specific measurement system. The classical phase-retrieval problem is to reconstruct the original signal from its $n$ spectral intensities $\left|F\left(\omega_{j}\right)\right|^{2}$. In particular, if the phases $\phi\left(\omega_{j}\right)$ are known, a standard procedure is to compute the $n$ discrete signal values

$$
f\left(t_{k}\right)=\frac{\Delta \omega}{2 \pi} \sum_{j=0}^{n-1}\left|F\left(\omega_{j}\right)\right| e^{\mathbf{i} \phi\left(\omega_{j}\right)+\mathbf{i} \omega_{j} t_{k}}
$$

where $t_{k}=\frac{2 \pi k}{n \Delta \omega}$. In this paper, we restrict our study to the discrete phase retrieval problem, and consider the reconstruction of the $n$ signal values $f\left(t_{k}\right)$ as the end goal ${ }^{1}$. For simplicity, we rescale time and frequency, such that $\Delta \omega=2 \pi / n$, and consequently $t_{k}=k$ for $k=1, \ldots, n$.

2) Trivial Ambiguities: Both $e^{\mathrm{i} \theta} f$ as well as circular time shifts of the original signal $f \in \mathbb{C}^{n}$ or its reflection, all yield the same spectral measurements. We thus say that the phase retrieval problem has a unique solution if all its solutions are of the above forms, which we consider as an equivalence class. Further, we remark that in our setting the refection ambiguity is ruled out by the additional interference measurements.

3) Signals With Limited Support: Given only $\left|F\left(\omega_{j}\right)\right|$, any phase vector $\left(\phi\left(\omega_{1}\right), \ldots, \phi\left(\omega_{n}\right)\right)$ is a valid solution to the phase retrieval problem. To determine a unique solution (up to trivial ambiguities), additional assumptions are required.

Since in many physical scenarios signals have an effective finite duration with a fast decay beyond it, a common assumption is that the continuous signal has a strict compact support $\left[t_{\text {init }}, t_{\text {init }}+\tau\right]$. Accordingly, for the discrete signal we assume that $f\left(t_{k}\right)=0$ for all $t_{k}<t_{\text {init }}$ or $t_{k}>t_{\text {init }}+\tau$. For 2-D signals, this assumption typically yields a unique solution to the phase retrieval problem [15]. In contrast, for a complex-valued or real-valued 1-D signal with a support of length $k$, there exist $2^{k}$ or $2^{k / 2}$ different solutions, respectively [15], [37]. Hence, even though the limited support assumption significantly reduces the set of solutions, it is insufficient to uniquely determine it in 1-D.

4) Vectorial Phase Retrieval: A natural question is thus whether there are physically realizable settings and accompanying assumptions that yield a unique solution to the 1-D phase retrieval problem. As we originally proposed in [22], the key motivation underlying our work is that in various common measurement schemes, it is possible to sample more than just the absolute spectral intensity of a single signal. In this paper, we consider a setting where one measures not only the spectral intensities $\left|F_{1}(\omega)\right|^{2}$ and $\left|F_{2}(\omega)\right|^{2}$, of

\footnotetext{
${ }^{1}$ Note that since $n$ and $\Delta \omega$ are finite, $f\left(t_{k}\right) \neq f_{c}\left(t_{k}\right)$, though the two values are close if $n \gg 1$ and $\Delta \omega \ll 1$ (in dimensionless units) [36].
} 
two signals $f_{1}(t)$ and $f_{2}(t)$, but also, in separate experiments, their interferences ${ }^{2}\left|F_{3}(\omega)\right|^{2}=\left|F_{1}(\omega)+F_{2}(\omega)\right|^{2}$ and $\left|F_{4}(\omega)\right|^{2}=\left|F_{1}(\omega)+\mathbf{i} F_{2}(\omega)\right|^{2}$. A physical example is short electromagnetic pulses [22], where $F_{1}(\omega)$ and $F_{2}(\omega)$ correspond to two polarization components of the electric field. Using a polarizer and a spectrometer, one can sample $\left|F_{1}(\omega)\right|^{2},\left|F_{2}(\omega)\right|^{2}$ and $\left|F_{1}(\omega)+F_{2}(\omega)\right|^{2}$. Adding a quarter wave-plate gives $\left|F_{1}(\omega)+\mathbf{i} F_{2}(\omega)\right|^{2}$.

This novel setup, which we denote as vectorial phase retrieval, leads to the following problem:

Vectorial Phase Retrieval Problem: Given (possibly noisy) measurements of $\left|F_{1}\left(\omega_{j}\right)\right|^{2},\left|F_{2}\left(\omega_{j}\right)\right|^{2},\left|F_{3}\left(\omega_{j}\right)\right|^{2}$ and $\left|F_{4}\left(\omega_{j}\right)\right|^{2}$ at the $n$ frequencies $\omega_{j}=j \Delta \omega$, reconstruct the two discrete signals $f_{1}$ and $f_{2}$.

Extending our initial work [22], we present a detailed study of the VPR problem. First, we show that for signals of limited support the spectral interference data is sufficient to determine a unique solution. Next, we study the effect of measurement noise and derive a novel algorithm for vectorial phase retrieval and a criterion to estimate the signal's support.

\section{Vectorial Phase Retrieval}

First, let us clearly state our assumptions:

1) The four spectra $\left|F_{1}(\omega)\right|^{2}, \quad\left|F_{2}(\omega)\right|^{2}, \quad\left|F_{3}(\omega)\right|^{2}$ and $\left|F_{4}(\omega)\right|^{2}$ are all sampled at the same finite number $n$ of equidistant frequencies $\omega_{j}=j \Delta \omega=2 \pi j / n$.

2) The two discrete signals, $f_{1}, f_{2} \in \mathbb{C}^{n}$ have exact but in general unknown and possibly different supports of sizes $\tau_{1}, \tau_{2}$. We assume there is a $\tau \geq \max \left(\tau_{1}, \tau_{2}\right)$ and an initial time $1 \leq t_{\text {init }} \leq n-\tau$ such that $f_{1, k}=f_{1}\left(t_{k}\right)=0$ and $f_{2, k}=f_{2}\left(t_{k}\right)=0$ for all $k<t_{\text {init }}$ and $k>t_{\text {init }}+\tau$.

3) The support size $\tau$ satisfies $\tau \leq n / 2$.

Remarks:

i) Recall that according to the Nyquist-Shannon theorem, for a continuous signal $f_{c}(t)$ of finite duration $\tau$, exact recovery from its sampled Fourier transform $F\left(\omega_{j}\right)$ is possible if and only if the sampling rate is higher than the Nyquist rate $\Delta \omega \leq \frac{\pi}{\tau}$ (see, e.g., chapter 8.2 in [36]). Since $\Delta \omega=2 \pi / n$, Assumption 3 requires that the sampling rate is higher than the Nyquist rate.

ii) If $\tau \leq n / 4$ (e.g., sampling at twice the Nyquist rate), then using recent results of Thakur [38], in the noise-free case, recovery is possible ${ }^{3}$ with only three measurements, $\left|F_{1}\right|,\left|F_{2}\right|$ and $\left|F_{1}+F_{2}\right|$.

Assumptions 1-3 do not guarantee a unique solution to the VPR problem. A trivial example is $f_{2}=c f_{1}$ for some $c \in \mathbb{C}$, which yields the classical 1-D problem, with many solutions. Intuitively, for the VPR problem to have a unique solution, the two signals $f_{1}$ and $f_{2}$ must be "independent" in some sense. As shown in the theorem below, the following definition captures exactly this requirement.

Definition: Two $n$-dimensional vectors $f_{1}, f_{2} \in \mathbb{C}^{n}$ are called spectrally independent if the two polynomials

\footnotetext{
${ }^{2}$ For simplicity, in this paper we consider spectral measurements of $f_{1}+f_{2}$ and of $f_{1}+\mathbf{i} f_{2}$. However, other or additional interferences of the form $f_{1}+$ $e^{\mathbf{i} \theta} f_{2}$ are possible and can easily be incorporated in our approach.

${ }^{3}$ The reason is that from these three measurements one may compute $\left|\operatorname{Im}\left\{F_{1} \bar{F}_{2}\right\}\right|$, which corresponds to a real-valued signal with support of size $2 \tau$, which may then be recovered via the methods of [38].
}

$P_{1}(z)=\sum_{k} f_{1}\left(t_{k}\right) z^{k}$ and $P_{2}(z)=\sum_{k} f_{2}\left(t_{k}\right) z^{k}$ have no common roots in the complex plane $z \in \mathbb{C}$.

The following theorem provides a necessary and sufficient condition for the VPR problem to have a unique solution.

Theorem 1: Let $f_{1}, f_{2} \in \mathbb{C}^{n}$ be two discrete signals that fulfill assumptions (1), (2) and (3). Up to trivial ambiguities (multiplication by $e^{\mathbf{i} \theta}$ and a circular time shift), the noise-free vectorial phase retrieval problem has a unique solution if and only if $f_{1}$ and $f_{2}$ are spectrally independent.

A proof of this theorem is given in the appendix. A sketch of this statement and its proof was first given by us in [22].

Remark: Note that almost any pair of arbitrary signals $f_{1}, f_{2}$ are spectrally independent, hence leading to a unique solution of the phase retrieval problem. More precisely, for fixed $f_{1} \in \mathbb{C}^{n}$, $f_{1} \neq 0$, the set of signals $f_{2} \in \mathbb{C}^{n}$ with unit norm $\left\|f_{2}\right\|=1$ such that $f_{1}, f_{2}$ are spectrally dependent is of measure zero.

\section{Vectorial Phase Retrieval in the Presence of Noise}

In any experimental system the spectral intensities are measured with some noise, and are thus inconsistent with assumption (2) of a finite support of length $\tau$. Namely, there are no spectral phases which, combined with the noisy spectra, result in finite support signals. Hence, the uniqueness guaranteed by Theorem 1 is not directly applicable. In the presence of noise, vectorial phase retrieval becomes a statistical problem. Common methods are maximizing the likelihood, or minimizing some convex proxy thereof, when the likelihood is non-convex. To this end, we first present an explicit modelling of the measurement noise.

\section{A. Noise Model}

Let $\left|\tilde{F}_{i}\left(\omega_{j}\right)\right|^{2}$ be the noisy measurements corresponding to $\left|F_{i}\left(\omega_{j}\right)\right|^{2}$, for $i=1, \ldots, 4$. While the exact relation between these two quantities depends on the specific experimental apparatus, a rather general model is that at frequency $\omega$,

$$
\left|\tilde{F}_{i}(\omega)\right|^{2}=\left|F_{i}(\omega)+\eta_{i}^{s}(\omega)\right|^{2}+\left|F_{i}(\omega)+\eta_{i}^{s}(\omega)\right| \eta_{i}^{s h}(\omega)+\eta_{i}^{d}(\omega)
$$

where $\eta_{i}^{s}(\omega)$ is background additive noise, $\eta_{i}^{d}(\omega)$ is the detector's noise independent of the signal (e.g., dark counts), and $\left|F_{i}(\omega)+\eta_{i}^{s}(\omega)\right| \eta_{i}^{s h}(\omega)$ is the detector's shot noise which is proportional to the signal's intensity. The detector's noise due to dark counts is always nonnegative, and in many practical cases of interest has relatively small variance. Its main effect can then be removed by a proper calibration. Hence, for simplicity, in this paper we assume that $\eta_{i}^{d}(\omega)=0$.

Next, since all signals are measured with the same equipment, we assume equal noise levels in all four measurements. Furthermore, we assume $\eta_{1 . .4}^{s}(\omega)$ are all independent and identically distributed (i.i.d.) complex-valued random variables, $\mathcal{C} \mathcal{N}\left(0, \sigma^{2}\right)$. As for the shot noises $\eta_{1 . .4}^{s h}(\omega)$, we assume they are all i.i.d. with zero mean and unit variance. In particular, noises at different frequencies are uncorrelated. This is indeed reasonable in many physical realizations where different spectral components are measured by separate detector elements, for example, as in a spectrometer.

\section{B. A Quadratic Functional for Vectorial Phase Retrieval}

Recall that VPR has a time shift ambiguity, where both $\left(f_{1}\left(t_{k}\right), f_{2}\left(t_{k}\right)\right)$ and $\left(f_{1}\left(t_{k}+t_{0} \bmod n\right), f_{2}\left(t_{k}+t_{0} \bmod n\right)\right)$ yield the same values $\left|F_{i}\left(\omega_{j}\right)\right|, i=1, \ldots, 4$. Hence, without 
loss of generality we may assume that the two signals are non-zero at the indices $\{1 \ldots \tau\}$ and vanish at the indices $\{\tau+1, \ldots, n\}$. At this stage we assume that $\tau$ is known, though later we discuss how to estimate it from the measured data.

The standard approach to estimate $f_{1}$ and $f_{2}$ is to maximize the likelihood $\mathcal{L}\left(\left|\tilde{F}_{i}\right| \mid f_{1}, f_{2}\right)$. Unfortunately, this is an intractable non-convex optimization problem. For a different set of measurements (multiple structured illuminations of a single signal), [29] proposed a semi-definite program as a convex relaxation of this likelihood. In principle, this approach is also applicable in our setting. In this paper, however, we propose an alternative approach. Instead of working with the unknown time domain values $f_{1,2}\left(t_{k}\right)$, we consider the unknown phases $x_{1}\left(\omega_{j}\right)=e^{\mathbf{i} \phi_{1}\left(\omega_{j}\right)}$ and $x_{2}\left(\omega_{j}\right)=e^{\mathbf{i} \phi_{2}\left(\omega_{j}\right)}$. This approach allows us to recover the phases by minimizing an appropriate quadratic functional, namely by solving a linear system of equations. We note that for a different set of noise-free measurements, [34] also work directly with the unknown phases, retrieving them via a more computationally intensive convex programming approach.

1) Limited Support: Let $\hat{x}_{1}, \hat{x}_{2} \in \mathbb{C}^{n}$ be some estimators of the phases. Then a corresponding estimate for the signal is

$$
\hat{f}_{1}\left(t_{k}\right)=\frac{1}{n} \sum_{j}\left|\tilde{F}_{1}\left(\omega_{j}\right)\right| \hat{x}_{1}\left(\omega_{j}\right) e^{\mathbf{i} \omega_{j} t_{k}}
$$

with a similar expression for $\hat{f}_{2}$. In particular, outside the support $[1, \tau]$ (which for the moment is assumed known), both $\hat{f}_{1}$ and $\hat{f}_{2}$ should be small since the actual signals vanish there. For $t_{k}>\tau$, we thus denote these residual values by

$$
R_{1,2}\left(t_{k}\right)=R_{1,2}\left(t_{k} \mid \hat{x}_{1}, \hat{x}_{2}\right)=\hat{f}_{1,2}\left(t_{k}\right) .
$$

In the ideal noise-free case, at the true phase vectors $x_{1}, x_{2}$, these residuals vanish exactly. In contrast, in the presence of noise, no phase vectors give zero residuals.

If these residuals $R_{1}\left(t_{k}\right)$ and $R_{2}\left(t_{k}\right)$ had a zero mean Gaussian distribution and were independent of each other and of the unknown signals, a sensible approach would be to search for phase vectors which minimize

$$
\sum_{t_{k}>\tau} \frac{\left|R_{1}\left(t_{k} \mid \hat{x}_{1}\right)\right|^{2}}{V_{1}}+\sum_{t_{k}>\tau} \frac{\left|R_{2}\left(t_{k} \mid \hat{x}_{2}\right)\right|^{2}}{V_{2}},
$$

where

$$
V_{1}=\mathbb{E}\left[\left|R_{1}\left(t_{k} \mid x_{1}\right)\right|^{2}\right], \quad V_{2}=\mathbb{E}\left[\left|R_{2}\left(t_{k} \mid x_{2}\right)\right|^{2}\right]
$$

are the variances of $R_{1,2}\left(t_{k} \mid x_{1}, x_{2}\right)$ respectively. Lemma 2 in the appendix shows that in the high SNR limit, the residuals $R_{1}$ and $R_{2}$ are weakly correlated and thus minimizing (5) is a sensible approach. Moreover, it shows that the variances of $R_{1,2}\left(t_{k}\right)$ are equal $\left(V_{1}=V_{2}\right)$ and independent of $k$, as implied implicitly in (6) above.

From a computational perspective, the key point is that the residuals $R_{1}$ and $R_{2}$ are linear in the unknown phase vectors $x_{1}$ and $x_{2}$. Thus, (5) is a quadratic (and hence convex) function of these variables.

\footnotetext{
${ }^{4}$ The phases are well defined only when $F_{1}\left(\omega_{j}\right)$ and $F_{2}\left(\omega_{j}\right)$ do not vanish. As discussed below, our approach works also when some of them vanish exactly or are very small.
}

2) Interference Information: The residuals $R_{1}$ and $R_{2}$ capture the support assumption on $f_{1}$ and $f_{2}$. To ensure a unique solution, we next consider the spectral interferences. In the absence of noise and suppressing the dependence on $\omega_{j}$,

$$
\begin{aligned}
\left|F_{3}\right|^{2} & =\left|F_{1}+F_{2}\right|^{2} \\
& =\left|F_{1}\right|^{2}+\left|F_{2}\right|^{2}+2\left|F_{1}\right|\left|F_{2}\right| \Re\left\{x_{1} \overline{x_{2}}\right\} \\
\left|F_{4}\right|^{2} & =\left|F_{1}+\mathbf{i} F_{2}\right|^{2} \\
& =\left|F_{1}\right|^{2}+\left|F_{2}\right|^{2}+2\left|F_{1}\right|\left|F_{2}\right| \Im\left\{x_{1} \overline{x_{2}}\right\} .
\end{aligned}
$$

Multiplying (8) by $\mathbf{i}$ and adding (7) gives the following linear relation between $x_{1}\left(\omega_{j}\right)$ and $x_{2}\left(\omega_{j}\right)$

$$
x_{1}\left(\omega_{j}\right)=x_{2}\left(\omega_{j}\right) G\left(\omega_{j}\right)
$$

where suppressing the dependence on $\omega_{j}$

$$
G=\frac{\left|F_{3}\right|^{2}+\mathbf{i}\left|F_{4}\right|^{2}-(1+\mathbf{i})\left(\left|F_{1}\right|^{2}+\left|F_{2}\right|^{2}\right)}{2\left|F_{1}\right|\left|F_{2}\right|} .
$$

In the noisy case we define the following residuals

$$
R_{3}\left(\omega_{j}\right)=R_{3}\left(\omega_{j} \mid \hat{x}_{1}, \hat{x}_{2}\right)=\hat{x}_{1}\left(\omega_{j}\right)-\hat{x}_{2}\left(\omega_{j}\right) \frac{\tilde{G}\left(\omega_{j}\right)}{\left|\tilde{G}\left(\omega_{j}\right)\right|},
$$

where, again suppressing the dependence on $\omega_{j}$

$$
\tilde{G}=\frac{\left|\tilde{F}_{3}\right|^{2}+\mathbf{i}\left|\tilde{F}_{4}\right|^{2}-(1+\mathbf{i})\left(\left|\tilde{F}_{1}\right|^{2}+\left|\tilde{F}_{2}\right|^{2}\right)}{2\left|\tilde{F}_{1}\right|\left|\tilde{F}_{2}\right|} .
$$

Obviously, $R_{3}$ is also linear in the phase vectors $\hat{x}_{1}, \hat{x}_{2}$. Moreover, at the true phase vectors, in the absence of noise, $R_{3}\left(\omega_{j}\right)=$ 0 . In the noisy case we view $\tilde{G} /|\tilde{G}|$ as an estimate of the relative phase between $x_{1}$ and $x_{2}$.

Lemma 3 in the appendix characterizes the statistical properties of $R_{3}$. It shows that at low noise levels, $R_{3}\left(\omega_{j}\right)$ are all approximately independent zero mean Gaussian random variables. Thus, a suitable quadratic functional is $\sum_{j=1}^{n} \frac{\left|R_{3}\left(\omega_{j}\right)\right|^{2}}{V_{3, j}}$, where $V_{3, j}=\mathbb{E}\left[\left|R_{3}\left(\omega_{j} \mid x_{1}, x_{2}\right)\right|^{2}\right]$ depends on the index $j$ and is explicitly given in (41).

Remark: If either $F_{1}\left(\omega_{j}\right)=0$ or $F_{2}\left(\omega_{j}\right)=0$, then $G\left(\omega_{j}\right)$ is clearly not well defined. The quantity $\tilde{G}\left(\omega_{j}\right)$ is then random and very large, $\mathcal{O}(1 / \sigma)$. While the equation for $R_{3}\left(\omega_{j}\right)$ is also random and meaningless, in this case $V_{3, j}$ is $\mathcal{O}\left(1 / \sigma^{2}\right)$, so the effect of this residual is strongly discounted. Moreover, if $F_{1}\left(\omega_{j}\right)$ is very small compared to $\sigma$ or even identically zero, then the reconstruction of $f_{1}$ is hardly affected by the value of the estimated phase $\hat{x}_{1}\left(\omega_{j}\right)$. In simple words, our algorithm, described below, is able to handle also a few small or vanishing Fourier coefficients.

3) Quadratic Functional: So far we defined quadratic functionals for the support of the two signals and for the interference. Lemma 4 shows that at low noise levels, $R_{3}$ is weakly correlated with $R_{1}$ and $R_{2}$, though unfortunately the correlations depend on the unknown signals. Neglecting these correlations, we propose the following $\tau$-dependent functional $Q_{\tau}$ by summing the three residuals,

$$
Q_{\tau}(\hat{\mathrm{x}})=\sum_{t_{k}>\tau} \frac{\left|R_{1}\left(t_{k}\right)\right|^{2}}{V_{1}}+\frac{\left|R_{2}\left(t_{k}\right)\right|^{2}}{V_{2}}+\sum_{j=1}^{n} \frac{\left|R_{3}\left(\omega_{j}\right)\right|^{2}}{V_{3, j}},
$$

where $\hat{\mathrm{x}}=\left(\hat{x}_{1}, \hat{x}_{2}\right) \in \mathbb{C}^{2 n}$ are the $2 n$ unknown phases. 
The minimizer of this functional is our proposed solution for the vectorial phase retrieval problem. The following theorem, proven in the appendix, justifies using $Q_{\tau}(\hat{\mathrm{x}})$ as a quality measure of a given guess for the unknown phases.

Theorem 2: Let $f_{1}, f_{2} \in \mathbb{C}^{n}$ be two spectrally independent signals that satisfy assumptions (1)-(3). For noise-free measurements, at the exact support size $\tau$, up to multiplication by a global constant, $\mathrm{x}$ is the unique minimizer of $Q_{\tau}$.

\section{An Algorithm for Vectorial Phase Retrieval}

Our proposed algorithm for VPR involves two steps. First, we scan the possible values $s$ for the unknown support, where for each $s$ we look for the minimizer of $Q_{s}(\mathrm{x})$ among all phase vectors $\mathrm{x}=\left(x_{1}\left(\omega_{1}\right), \ldots, x_{1}\left(\omega_{n}\right), x_{2}\left(\omega_{1}\right), \ldots, x_{2}\left(\omega_{n}\right)\right) \in \mathbb{C}^{2 n}$. Second, we estimate the support $\hat{\tau}$, by solving an appropriate model selection problem.

\section{A. Finding the Minimizer of $Q_{s}(\mathrm{x})$}

First, assuming a support of size $s$, our goal is to minimize $Q_{s}(\mathrm{x})$ over the set of phase vectors $(|\mathrm{x}(j)|=1$ for all $j)$.

To this end, we represent $Q_{s}$ explicitly as a quadratic form. Recall that for a support size $s$, the length of $R_{1}$ and $R_{2}$ is $n-s$, whereas $R_{3}$ is of length $n$, independent of $s$. Hence, the quadratic functional $Q_{s}$ can be written as

$$
Q_{s}(\mathrm{x})=\mathrm{x}^{H} \mathcal{A}_{s}^{H} \mathcal{A}_{s} \mathrm{x}
$$

where the matrix $\mathcal{A}_{s}$, of size $(3 n-2 s) \times 2 n$, is given by

$$
\left(\mathcal{A}_{s}\right)_{k, j}= \begin{cases}\frac{\left|\tilde{F}_{1}\left(\omega_{j}\right)\right|}{\sqrt{V_{1}}} e^{\mathbf{i} \omega_{j} t_{k}} & k \leq n-s, j \leq n \\ \frac{\left|\tilde{F}_{2}\left(\omega_{j-n}\right)\right|}{\sqrt{V_{2}}} e^{\mathbf{i} \omega_{j-n} t_{k-n+s}} & n-s<k \leq 2(n-s), j>n \\ \frac{1}{\sqrt{V_{3, j}}} & k-2(n-s)=j \\ \frac{\tilde{G}\left(\omega_{j-n}\right)}{\left|\tilde{G}\left(\omega_{j-n}\right)\right| \sqrt{V_{3, j-n}}} & k-2(n-s)=j-n \\ 0 & \text { otherwise. }\end{cases}
$$

Using Lagrange multipliers for the $2 n$ phase constraints gives the following unconstrained minimization problem

$$
\min _{\mathrm{x}, \lambda_{j}}\left\{Q_{s}(\mathrm{x})+\sum_{j=1}^{2 n} \lambda_{j}\left(|\mathrm{x}(j)|^{2}-1\right)\right\} \text {. }
$$

This minimization is equivalent to the "multivariate eigenvalue problem", which is non-convex, but several iterative algorithms to solve it were derived [39]-[41].

In this paper we suggest a relaxation of these constraints that leads to a convex optimization problem. First note that the reconstruction problem has a trivial ambiguity: $Q_{s}(\mathrm{x})=Q_{s}\left(e^{\mathrm{i} \theta} \mathrm{x}\right)$ for any real $\theta$. Thus, without loss of generality, we can choose the solution $\mathrm{x}$ such that $\mathrm{x}(v)=-1$ for some specific index $v$. Using (14), the quadratic functional $Q_{s}$ can then be written as:

$$
Q_{s}(\mathrm{x})=\mathrm{x}^{H} \mathcal{A}_{s}^{H} \mathcal{A}_{s} \mathrm{x}=\left\|\left(\mathcal{A}_{s}\right)_{-v} \mathrm{x}_{-v}-a_{v}\right\|^{2}
$$

where $\left(\mathcal{A}_{s}\right)_{-v}$ is the matrix $\mathcal{A}_{s}$ without the $v^{t h}$ column, $a_{v}$ is the $v^{t h}$ column of $\mathcal{A}_{s}$ and $\mathrm{x}_{-v}$ is the phase vector $\mathrm{x}$ without its $v^{t h}$ entry.

Minimizing $\left\|\left(\mathcal{A}_{s}\right)_{-v} \mathrm{x}_{-v}-a_{v}\right\|^{2}$ under the constraint that $\mathrm{x}_{-v}$ is a phase vector is still a non-convex optimization problem.
In our approach, we drop the phase constraints, and minimize (17) without any constraints at all. This amounts to solving a linear system of equations. Since $\sqrt{V_{1}}, \sqrt{V_{2}}$ and $\sqrt{V_{3, j}}$ are all proportional to $\left(1 / 4+\sigma^{2}\right)^{1 / 2}$ (see Lemmas 2 and 3 in the Appendix), so are all entries of $\mathcal{A}_{s}$. Hence, knowing $\sigma$ is not needed for minimizing $\left\|\left(\mathcal{A}_{s}\right)_{-v} \mathrm{x}_{-v}-a_{v}\right\|^{2}$.

There are several options for the index $v$. One that worked well in practice is to choose the column $a_{v}$ with maximal norm, as this is the most dominant column in the matrix $\mathcal{A}_{s}$.

For noise free measurements corresponding to signals with an exact support $\tau$, when $s=\tau$ the true phase vector gives $\left\|\left(\mathcal{A}_{\tau}\right)_{-v} \mathrm{x}_{-v}-a_{v}\right\|^{2}=0$, and is thus a minimizer of $Q_{\tau}$. Theorem 2 ensures that this is the unique minimizer, hence in the noise-free case our algorithm retrieves the correct solution.

In the presence of noise, for any value of $s$ the minimizer $\tilde{\mathrm{x}}_{s}$ of $\left\|\left(\mathcal{A}_{s}\right)_{-v} \mathrm{x}_{-v}-a_{v}\right\|^{2}$ is not, in general, a phase vector. Assuming a support of length $s$, our proposed solution is thus its following projection into a phase vector,

$$
\hat{\mathrm{x}}_{s}(j)=\frac{\tilde{\mathrm{x}}_{s}(j)}{\left|\tilde{\mathrm{x}}_{s}(j)\right|} \text {. }
$$

Remark: There are several alternative approaches to relax the non-convex problem of minimizing $Q_{s}(\mathrm{x})$ over the set of phase vectors. For example, replacing the $2 n$ constraints of (16) by a single one of the form $\sum|\mathrm{x}(j)|^{2} W(j)=\sum W(j)$ for non-negative weights $W(j)$ gives a convex generalized eigenvalue problem. If $W(j)=1$ it reduces to the smallest eigenvalue of $Q_{s}$, whereas if $W(j)=0$ for $j \neq v$ and $W(v)=1$ it recovers (17). Numerically, we found our relaxation to yield slightly smaller errors than those of the smallest eigenvector. A theoretical study of these relaxations is an interesting topic beyond the scope of this manuscript. Finally, we remark that similar relaxations appear in a different problem of angular synchronization, as studied by Singer [42].

\section{B. Estimating the Support Size $\tau$}

The remaining problem is to estimate the signal's support size $\tau$. Let us first consider the noise-free case with signals $f_{1}, f_{2}$ that have an exact limited support. To estimate $\tau$, we scan all possible supports, and separately optimize $Q_{s}$ for each $s$. Clearly, at $s=\tau$, the correct phase vector is the unique vector that gives $Q_{\tau}(\mathrm{x})=0$. Hence, $\hat{\mathrm{x}}_{\tau}=\tilde{\mathrm{x}}_{\tau}=\mathrm{x}$.

For $s<\tau$, no phase vector x gives $R_{1}\left(t_{k}\right)=R_{2}\left(t_{k}\right)=0$ for all $t_{k}>s$. Hence for any phase vector $\hat{\mathrm{x}}, Q_{s}(\hat{\mathrm{x}})>0$. In particular $Q_{s}\left(\hat{\mathrm{x}}_{s}\right)>Q_{\tau}\left(\hat{\mathrm{x}}_{\tau}\right)=0$.

For $s>\tau$, both the original signals with support $\{1, \ldots, \tau\}$ as well as small time shifts of them, with support $\{w, \ldots, \tau+w\}$ are identically zero for $t>s$, as long as $w \leq s-\tau$. Thus, even for spectrally independent signals, there are several distinct phase vectors $\mathrm{x}$ for which $Q_{s}(\mathrm{x})=0$. That is, the resulting linear system has an infinite number of solutions residing in an affine subspace of $\mathbb{C}^{2 n}$. With probability one, the output of the numerical algorithm minimizing $\left\|\left(\mathcal{A}_{s}\right)_{-v} \mathrm{x}_{-v}-a_{v}\right\|^{2}$ is not a phase vector. Hence, upon its projection to a phase vector via (18), $\hat{\mathrm{x}}_{s} \neq \mathrm{x}$ and $Q_{s}\left(\hat{\mathrm{x}}_{s}\right)>0$ for any $s>\tau$.

Thus, in the noise-free case, we can recover the true support by finding the values of $s$ for which $Q_{s}\left(\tilde{\mathrm{x}}_{s}\right)$ is minimal.

Next, consider the case of noisy measurements. For simplicity, we assume $\sigma$ to be known. For any value of $s$, there are no phase vectors such that $Q_{s}(\hat{\mathrm{x}})=0$. Nonetheless, as the 
TABLE I

Algorithm For Vectorial PhASE RETRIEVAL

Input: $\left|\tilde{F}_{1}\left(\omega_{j}\right)\right|^{2},\left|\tilde{F}_{2}\left(\omega_{j}\right)\right|^{2},\left|\tilde{F}_{3}\left(\omega_{j}\right)\right|^{2},\left|\tilde{F}_{4}\left(\omega_{j}\right)\right|^{2}, \tau_{\min }, \tau_{\max }, \sigma$ Algorithm:

1: [Scan support size] For $s=\tau_{\min }$ to $\tau_{\max }$ do: a: Compute the minimizer $\hat{\mathrm{x}}_{s}$ of $\left\|\left(\mathcal{A}_{s}\right)_{-v} \mathrm{x}_{-v}-a_{v}\right\|^{2}$.

b: [Project to phase vector] $\tilde{\mathrm{x}}_{s}(j)=\frac{\hat{\mathrm{x}}_{s}(j)}{\left|\hat{\mathrm{x}}_{s}(j)\right|}$, for $j=1 \ldots 2 n$

c: Compute time residual $r(s)$ given in Eq.(20).

2: Estimate the support by $\hat{\tau}=\arg \min _{s} r(s)$, and the phase by $\tilde{\mathrm{x}}=\tilde{\mathrm{x}}_{\tilde{\tau}}$. 3: Reconstruct the signals:

$$
\begin{aligned}
& \hat{f}_{1}\left(t_{k}\right)= \begin{cases}\frac{1}{n} \sum_{j=1}^{n}\left|\tilde{F}_{1}\left(\omega_{j}\right)\right| \tilde{\mathrm{x}}(j) e^{\mathrm{i} \omega_{j} t_{k}} & t_{k} \leqslant \hat{\tau} \\
0 & t_{k}>\hat{\tau}\end{cases} \\
& \hat{f}_{2}\left(t_{k}\right)= \begin{cases}\frac{1}{n} \sum_{j=1}^{n}\left|\tilde{F}_{2}\left(\omega_{j}\right)\right| \tilde{\mathrm{x}}(j+n) e^{\mathrm{i} \omega_{j} t_{k}} & t_{k} \leqslant \hat{\tau} \\
0 & t_{k}>\hat{\tau}\end{cases}
\end{aligned}
$$

Output: $\hat{f}_{1}, \hat{f}_{2}$.

minimizer of $\left\|\left(\mathcal{A}_{s}\right)_{-v} \mathrm{x}_{-v}-a_{v}\right\|^{2}$ is a continuous function of the measurements, for low noise levels, at $s=\tau$ we expect $\hat{\mathrm{x}}_{\tau}$ to be close to $\mathrm{x}$, and consequently $Q_{\tau}\left(\hat{\mathrm{x}}_{\tau}\right)$ to be close to zero. However, since the length of the two residuals $R_{1}$ and $R_{2}$ decreases with $s$, estimating $\tau$ by $\arg \min _{s} Q_{s}\left(\hat{\mathrm{x}}_{s}\right)$ may yield an incorrect support size and suboptimal reconstruction.

From a statistical point of view, estimating the support size $\tau$ in the presence of noise can be formulated as a nested model selection problem. Since the support size affects mainly the two residuals $R_{1}$ and $R_{2}$, in order to estimate it we only consider them and disregard the residual $R_{3}$.

To motivate our proposed model selection criterion, note that assuming a support $s$, the length of the residual vectors $R_{1,2}$ is $(n-s)$ and in particular decreases with $s$. Moreover, according to Lemma 2, when $R_{1}$ and $R_{2}$ are evaluated with the correct phase vector $\mathrm{x}$, at low noise levels,

$$
\mathbb{E}\left[\sum_{t_{k}>s}\left|R_{1,2}\left(t_{k} \mid \mathrm{x}\right)\right|^{2}\right]=(n-s) \frac{1}{n}\left(\frac{1}{4}+\frac{\sigma^{2}}{2}\right) .
$$

Similar to other model selection criteria, we construct a functional with a data fit term and a penalty term with a penalty factor $C$,

$r(s)=\left(\sum_{t_{k}>s}\left|R_{1}\left(t_{k} \mid \tilde{\mathrm{x}}\right)\right|^{2}+\left|R_{2}\left(t_{k} \mid \tilde{\mathrm{x}}\right)\right|^{2}\right)+C \frac{2 s}{n}\left(\frac{1}{4}+\frac{\sigma^{2}}{2}\right)$.

Our proposed $\hat{\tau}$ is then

$$
\hat{\tau}=\arg \min _{s} r(s)
$$

The penalty term in (20) depends on the constant $C$. In light of (19), $C$ should be at least 1 . In our simulations we used $C=2$, though our recovered $\hat{\tau}$ is quite robust to the exact value of $C$. A detailed study of this model selection problem is an interesting topic beyond the scope of this paper.

In summary, Table I describes our vectorial phase retrieval algorithm. It assumes that the support is in some range $\tau \in$ $\left[\tau_{\min }, \tau_{\max }\right]$. In the absence of such prior information, $\tau_{\min }=1$ and $\tau_{\max }=n / 2$. Note that when reconstructing the signals, consistent with our assumption of a support $\hat{\tau}$, we set to zero all values of $\hat{f}_{1,2}$ beyond $\hat{\tau}$.

\section{Computational Complexity}

To analyze the computational complexity of our algorithm, consider the solution of (17) for a given $s$, via the set of linear equations $\left(\mathcal{A}_{s}^{H}\right)_{-v}(\mathcal{A})_{-v} \mathrm{x}_{-v}=\left(\mathcal{A}_{s}^{H}\right)_{-v} a_{v}$. Calculating $\left(\mathcal{A}_{s}^{H}\right)_{-v}(\mathcal{A})_{-v}$ and solving the set of linear equations both require $\mathcal{O}\left(n^{3}\right)$ operations. The number of times such equations have to be solved depends on our prior knowledge of $\tau$. If it is roughly known (e.g., when it is set by the experimental setup) then only a limited scan over $s$ (if at all) is required, and the overall complexity is $\mathcal{O}\left(n^{3}\right)$. In the worst case, $\tau_{\min }=1, \tau_{\max }=n / 2$ and the overall complexity is $\mathcal{O}\left(n^{4}\right)$.

In comparison, the computational complexity of the SDP algorithm of [34] adapted to our setup and with a known support is either $\mathcal{O}\left(n^{3.5} \log \frac{1}{\epsilon}\right)$ or $\mathcal{O}\left(n^{3} \frac{1}{\epsilon}\right)$, where $\epsilon$ is the accuracy of the SDP. Numerically we compared a Matlab implementation of our algorithm to that of [34] (available at the authors website). For a relatively short signal of length $n=200$, our algorithm was not only slightly more accurate, but also about 1000 times faster (with a running time of $10^{-2} \mathrm{sec}$. compared to about 10 sec. for the SDP approach, both codes in Matlab running on a standard desktop PC).

\section{Simulations}

We illustrate our reconstruction algorithm by simulations on either strictly supported signals or on exponentially decaying signals that have no strict support, in both cases with and without measurement noise. In the presence of noise, for simplicity we assume the noise level $\sigma$ is known.

We measure the quality of a reconstruction $\hat{f}$ by a normalized mean squared error (MSE), which is invariant to trivial ambiguities of time shifts and multiplication by a global phase,

$$
\mathrm{MSE}=\min _{t_{\text {init }}, \theta} \frac{\sum_{k} \mid e^{\mathbf{i} \theta \hat{f}\left(\left(t_{k}-t_{\text {init }}\right) \bmod n\right)-\left.f\left(t_{k}\right)\right|^{2}}}{\sum_{k}\left|f\left(t_{k}\right)\right|^{2}} .
$$

\section{A. Reconstruction in Absence of Noise}

First, we show that our algorithm is able to perfectly recover signals with finite support from noise free spectral measurements. Fig. 1(a) shows the signal $f_{1}$ of length $n=200$, with a strict support of length $\tau=58$ and its reconstruction whose MSE is nearly zero $\left(10^{-26}\right)$. The second signal $f_{2}$ had the same support and a similar reconstruction quality. Fig. 1(b) shows that $r(s)$ attains its minimum at the exact support length $s=\tau=58$, with a minimal value that is about 20 orders of magnitude lower than $r(s)$ for $s \neq \tau$.

Next, we show that our algorithm is also able to reconstruct signals that have no strict support. To this end, consider the following complex valued exponentially decaying signals,

$$
\begin{aligned}
& f_{1}(t)=C_{1}\left(t^{2} e^{-\left(t-t_{0}\right)^{2} / \sigma_{0}^{2}}+\mathbf{i} t^{2} e^{-\left(t-t_{1}\right)^{2} / \sigma_{1}^{2}}\right) \\
& f_{2}(t)=C_{2}\left(t^{2} e^{-\left(t-t_{0}\right)^{2} / \sigma_{0}^{2}}+0.3 \mathbf{i} t^{2} e^{-\left(t-t_{1}\right)^{2} / \sigma_{1}^{2}}\right)
\end{aligned}
$$

where $t \in[-200,0], t_{0}=-20, t_{1}=-40, \sigma_{0}=70, \sigma_{1}=20$, and $C_{1}$ and $C_{2}$ are determined such that $\left\|f_{1}\right\|=\left\|f_{2}\right\|=1$.

Fig. 2 shows the absolute values of $f_{1}(t)$ and of its reconstruction from noiseless measurements, as well as the residual 


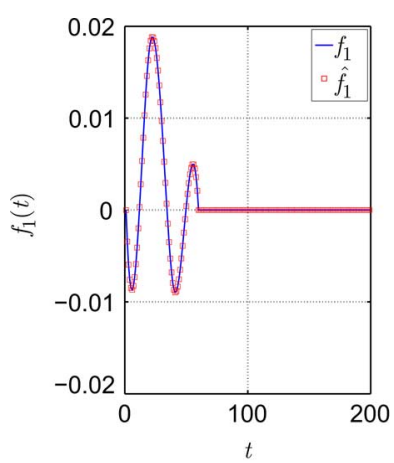

(a)

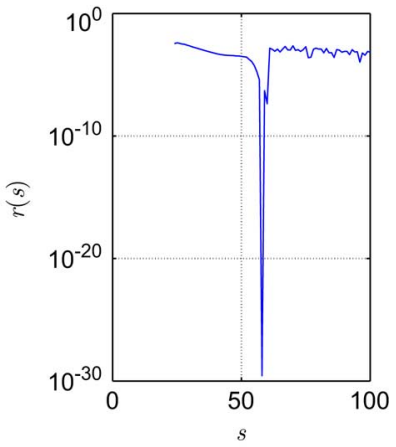

(b)
Fig. 1. Reconstruction of signals with finite support from noise free measurements. (a) Signal $f_{1}$ and its reconstruction $\hat{f}_{1}$. (b) $r(s)$ versus assumed support size $s$.
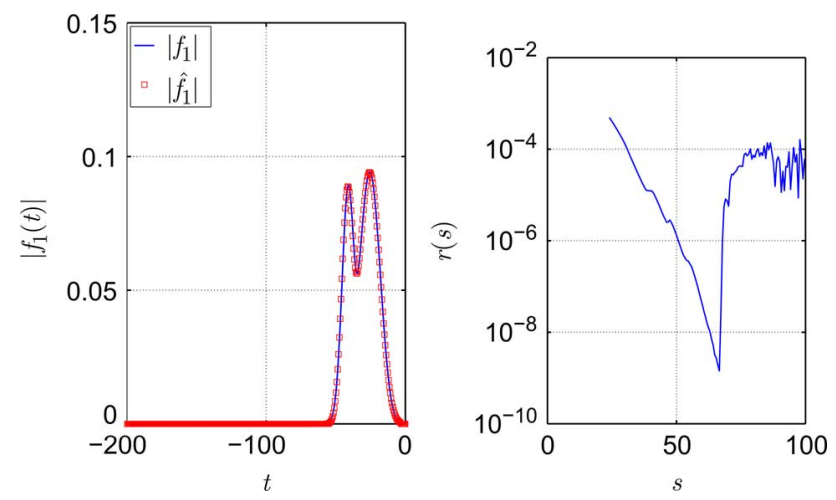

Fig. 2. Reconstruction of exponentially decaying signals from noise free measurements: left panel: the absolute of the complex valued signal $f_{1}$ of (23) and its reconstruction, $\hat{f}_{1}(t)$ (also in absolute value); right panel: the residual $r(s)$ versus assumed support length $s$.

$r(s)$. Even though both signals do not have a strict limited support, $r(s)$ has a clear minimum at $s=66$, though its minimal value there $\left(\approx 10^{-9}\right)$ is much higher compared to the case of strict limited support signals. Consequently, the reconstruction MSE is about $4 \times 10^{-4}$ for both $f_{1}$ and $f_{2}$.

\section{B. Reconstruction in Presence of Noise}

Next we show that our algorithm can accurately recover the signals even in the presence of noise. For clarity, we present results where only background noise is present (neglecting the effect of shot noise). This corresponds to either a single-shot or classical light experiment, away from the single photon regime. We note that our algorithm performed well also in the presence of shot noise, though these results are omitted due to space constraints.

Fig. 3 shows the reconstruction $\hat{f}_{1}$ of the signal $f_{1}$ of Fig. 1, and the residual $r(s)$ at a noise level $\sigma=0.01$. This corresponds to $\mathrm{SNR}=20 \log _{10} \frac{\left\|F_{1,2}\right\|}{\sqrt{\boldsymbol{n}} \sigma}=17 d B$. At this noise level, the estimated support size is still the correct one, $\hat{\tau}=58$.

Figs. 4(a), 4(b) show, on a logarithmic scale, MSE vs. noise level, averaged over 250 independent noise realizations at each noise level, for both signals with a limited support or with an exponential decay. In addition, we compared our algorithm to the one we previously suggested in [22], which lacked a detailed statistical analysis and justification. As the figure shows, the new algorithm performs significantly better.
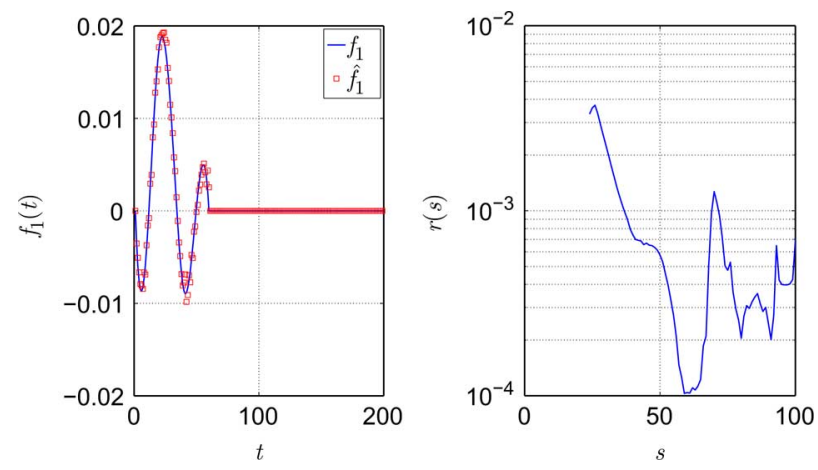

Fig. 3. Reconstruction in presence of noise: left panel: $f_{1}(t)$ and the real part of its (complex valued) reconstruction, $\hat{f}_{1}(t)$, at a noise level of $\sigma=0.01$; right panel: the residual $r(s)$.

Finally, Fig. 5 shows the estimated support size as a function of the noise level. As expected, for exponentially decaying signals, the higher the noise level, the smaller the estimated support size, since at high noise levels it is difficult to resolve the fine details of signals near their tails.

\section{Reconstruction Breakdown at High Noise Levels}

At high noise levels, several mechanisms may lead to poor signal reconstructions: (i) inaccurate estimation of the support, (ii) inaccurate phase estimation, and (iii) use of the noisy $\left|\tilde{F}\left(\omega_{j}\right)\right|$ to reconstruct the signal. To check which of these mechanisms is the dominant one, we present in Fig. 4(c) the MSE of four different methods vs. noise level: (i) our algorithm with its estimated support, (ii) our algorithm with the exact support, (iii) reconstruction with the estimated phase $\hat{x}$ but with the noise-free spectra $\left|F\left(\omega_{j}\right)\right|$, (iv) reconstruction with the exact phase $\mathbf{x}$ but with the noisy spectra $\left|\tilde{F}\left(\omega_{j}\right)\right|$.

First, note that the need to estimate $\tau$ introduces a negligible error. Our model selection criterion estimates the support quite well, and prior information about the exact support hardly improves our reconstruction.

Second, for low noise levels, most of the error is due to inaccuracies in the phase estimation. In contrast, at high noise levels, the main source of error is the noise in the measured amplitudes - even in case of successful phase reconstruction, the noise in $|\tilde{F}(\omega)|$ would still lead to a large error in $\|\hat{f}-f\|$.

These results suggest several possible future improvements. For example, one option is to denoise the noisy amplitude measurements prior to signal reconstruction. Another is to jointly estimate both the phase and the underlying amplitudes.

\section{Numerical Stability to Noise}

For simplicity we now study the stability to noise for signals with a strict support of known length $\tau$. Our algorithm boils down to a minimization problem which by our convex relaxation reduces to solving a set of linear equations. In the noise-free case this solution coincides with the eigenvector of smallest eigenvalue $\lambda_{1}=0$ of $Q_{\tau}$. Measurement noise perturbs all entries in $Q_{\tau}$ and hence also the solution. To leading order, its stability to noise depends primarily on the spectral gap to the next smallest eigenvalue $\lambda_{2}\left(Q_{\tau}\right)$.

We illustrate this dependency in Fig. 6(a). For this figure, we generated 1000 pairs of complex valued random signals $f_{1}, f_{2}$ of length $n=60$ with support length $\tau=n / 2$ and with i.i.d. $\mathbb{C} \mathcal{N}(0,1)$ entries. Next, we normalized each signal to unit norm 


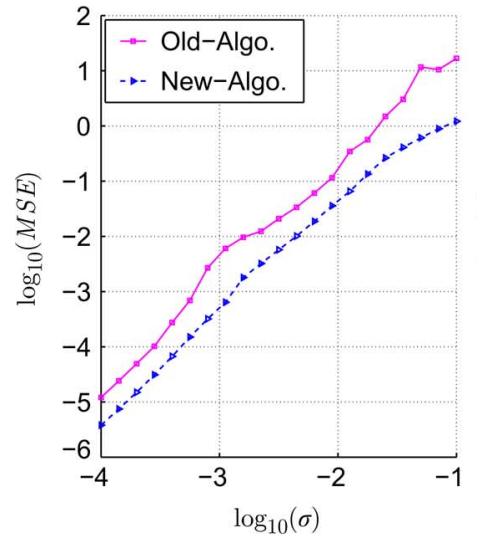

(a)

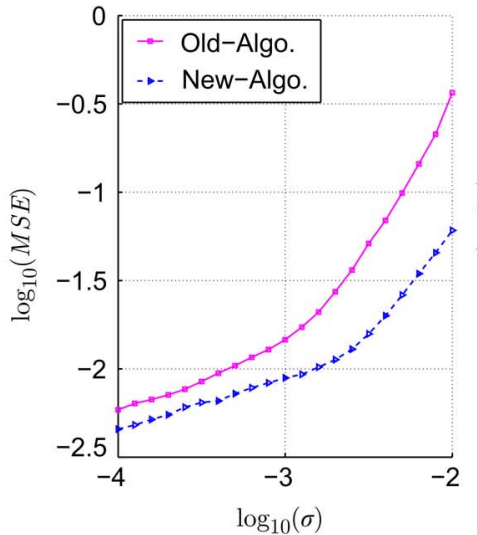

(b)

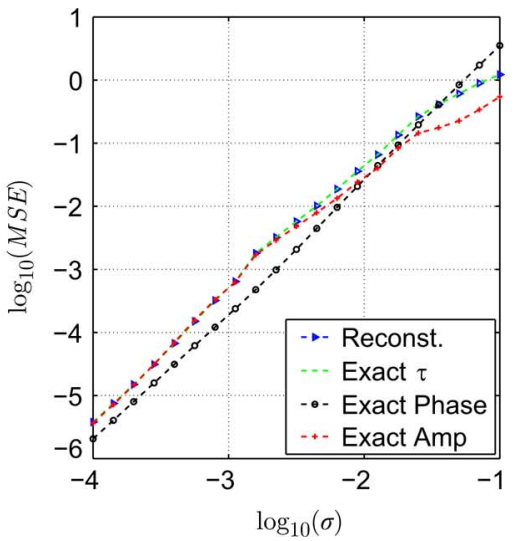

(c)

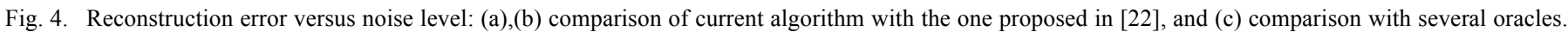
(a) Compact support signals. (b) Exponentially decaying signals. (c) Error of several oracle algorithms.
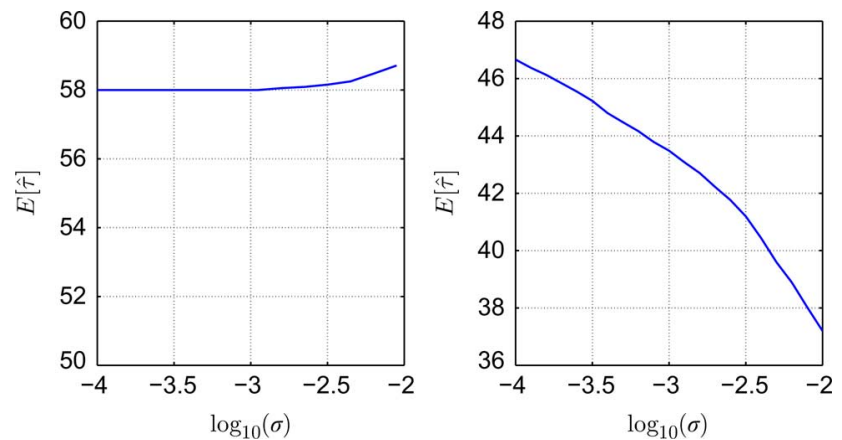

Fig. 5. $\mathbb{E}[\hat{\tau}]$ versus noise level, for supported signals (left) and for signals with exponential decay (right).

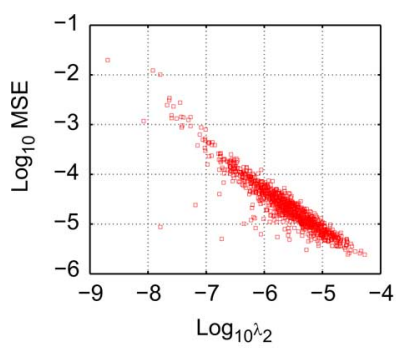

(a)

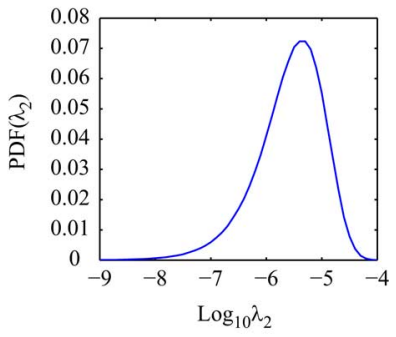

(b)
Fig. 6. Reconstruction stability to noise: (a) reconstruction MSE versus $\lambda_{2}\left(Q_{\tau}\right)$ and (b) prob. density of $\lambda_{2}\left(Q_{\tau}\right)$.

and computed $\lambda_{2}\left(Q_{\tau}\right)$. Finally, we measured the averaged MSE over 50 noise realizations at $\sigma=10^{-3}$. Fig. 6(a) compares the reconstructed MSE to $\lambda_{2}\left(Q_{\tau}\right)$. As can be seen, the spectral gap bounds the MSE.

Note that the spectral gap depends on the specific signals $f_{1}$ and $f_{2}$. A simple corollary of Thm. 2 is that the spectral gap is zero if and only if the two signals are spectrally dependent. Fig. 6(b) shows the probability density function of $\lambda_{2}\left(Q_{\tau}\right)$ estimated from $10^{6}$ pairs of random signals generated as described above. It suggests that the probability of an extremely small value of $\lambda_{2}$ (and hence potentially unstable reconstruction) is very low. A detailed study of our algorithm stability to noise is an interesting question for future research.

\section{SUMmARY AND DiscusSiON}

In this paper we presented a novel framework, along with accompanying theory and an efficient algorithm, for reconstruction of 1-D signals from modulus measurements of their Fourier coefficients, and of the Fourier coefficients of their interferences. Our work is a first step, which raises several theoretical and practical questions.

On the theoretical front, three open questions of interest are i) a theoretical guarantee of stability to noise; ii) a Cramer-Rao lower bound on the possible reconstruction error in VPR; and iii) an expression for the breakdown point - the noise level at which vectorial phase retrieval is not feasible from an information point of view.

On the computational front, our algorithm may be improved in several ways. For example, our relaxation of the phase constraints may be replaced by solving (non-convex) multivariate eigenvalue problems. Similarly, it might be possible to improve the reconstruction by taking into account additional knowledge about the signals, such as their sparsity in a given basis, or explicit knowledge, from physical considerations, about the form of their time decay. Also, the speed of our algorithm may be improved via multiscale methods.

Finally, while in this paper we focused on the 1-D case, vectorial phase retrieval can be extended to 2-D images, hence introducing a new class of computationally efficient imaging phase retrieval methods.

\section{APPENDIX I}

Proof of Theorem 1: To prove uniqueness of the noisefree VPR problem it is useful to first review the ambiguity of standard phase retrieval for a single 1-D signal $f \in \mathbb{C}^{n}$.

Following [15], we make a change of variables $z=e^{-\mathbf{i} \omega}$, known as the $z$-transform. In the new variable $z$, the discrete FT, $F(\omega)$, is simply a polynomial of degree at most $n$,

$$
E(z)=F(\omega)=\sum_{k} f\left(t_{k}\right) z^{k}
$$

We decompose this polynomial into its $n$ roots,

$$
E(z)=c \prod_{j}\left(z-z_{j}\right)
$$

where $c \in \mathbb{C}$ is some normalization constant. 
The spectral measurements can thus be written as

$$
|E(z)|^{2}=E(z) \overline{E(z)}=|c|^{2} \prod_{j}\left(z-z_{j}\right)\left(\bar{z}-\bar{z}_{j}\right) .
$$

For $|z|=1$ and $z_{j} \neq 0$ it holds that $5 \bar{z}-\bar{z}_{j}=-\frac{\overline{z_{j}}}{z}\left(z-\bar{z}_{j}^{-1}\right)$ Hence, for all $|z|=1$, and specifically at the $n$ measurement points,

$$
z^{n}|E(z)|^{2}=(-1)^{n}|c|^{2} \prod_{j} \bar{z}_{j} \prod_{j}\left(z-z_{j}\right)\left(z-\bar{z}_{j}^{-1}\right)
$$

is nothing but a polynomial of degree at most $2 n$.

With no prior knowledge about the signal, the spectral phase is unrecoverable: We have only $n$ observations $\left|E\left(e^{\mathbf{i} \omega_{j}}\right)\right|^{2}$ of a polynomial of degree $2 n$. If, however, $f$ has a support of length $k \leq n / 2$, meaning $f_{j}=0$ for all $j>k$, then $|E(z)|^{2}$ has degree at most $2 k \leq n$. The $n$ observations $\left|E\left(e^{\mathrm{i} \omega_{j}}\right)\right|^{2}=\left|F\left(\omega_{j}\right)\right|^{2}$ uniquely determine its $2 k$ roots $\left\{z_{j}, 1 / \bar{z}_{j}\right\}_{j=1}^{k}$. Nevertheless, the limited support constraint does not fully remove the ambiguity. To recover $f$, only one root from each pair, $z_{j}$ and $1 / \bar{z}_{j}$, should be chosen. This is the well known ambiguity of the 1-D phase retrieval [15], with $2^{k}$ different valid solutions.

Now let us analyze the uniqueness of the VPR problem. Let $f_{1}, f_{2}$ be the two signals, both with support of length $k \leq n / 2$, and let $\left\{z_{1 j}, 1 / \bar{z}_{1 j}\right\}$ and $\left\{z_{2 j}, 1 / \bar{z}_{2 j}\right\}$ be the $2 k$ roots of the two polynomials $\left|E_{1}(z)\right|^{2}$ and $\left|E_{2}(z)\right|^{2}$, respectively.

The key to uniqueness in vectorial phase retrieval lies in the additional spectral interference measurements. These allow recovery of the relative phase between the Fourier coefficients of the two signals. Indeed, suppressing the dependence on $z$,

$$
\frac{1}{2}\left(\left|E_{1}+E_{2}\right|^{2}+\mathbf{i}\left|E_{1}+\mathbf{i} E_{2}\right|^{2}-(1+\mathbf{i})\left(\left|E_{1}\right|^{2}+\left|E_{2}\right|^{2}\right)\right)=E_{1} \bar{E}_{2}
$$

Thus, in vectorial phase retrieval we also know $n$ values of $E_{1}(z) \bar{E}_{2}(z)$. Note that at $|z|=1$ this function is equal to a polynomial with the following factorization,

$$
z^{n} E_{1}(z) \overline{E_{2}(z)}=C \prod_{j}\left(z-z_{1 j}\right)\left(z-\bar{z}_{2 j}^{-1}\right) .
$$

Comparing the roots of $\left|E_{1}(z)\right|^{2}$ and $\left|E_{2}(z)\right|^{2}$ to those of (27), the ambiguity of each pair of roots can be resolved: The roots of $E_{1}(z)$ are those that are common to $\left|E_{1}(z)\right|^{2}$ and $E_{1}(z) \overline{E_{2}(z)}$, and similarly for $E_{2}(z)$. The requirement that $f_{1}$ and $f_{2}$ are spectrally independent allows for such a unique identification and is thus a sufficient condition for uniqueness.

To show that spectral independence is a necessary condition, consider $f_{1}, f_{2}$ which are spectrally dependent, with $E_{1}(z)$ and $E_{2}(z)$ sharing a common root $z_{k}$. Consider the two modified signals $\check{f}_{1}, \check{f}_{2}$ which correspond to $\check{E}_{1}(z)=\frac{\left(z-1 / \overline{z_{k}}\right) E_{1}(z)}{\left(z-z_{k}\right)}$ and $\check{E}_{2}(z)=\frac{\left(z-1 / \overline{z_{k}}\right) E_{2}(z)}{\left(z-z_{k}\right)}$. Clearly $\check{f}_{1}, \check{f}_{2}$ have the same support as $f_{1}, f_{2}$ as the degree of the corresponding polynomials is the same. Their Fourier coefficients have the same amplitude, as on the unite circle $\left|E_{1}(z)\right|^{2}=\left|\check{E}_{1}(z)\right|^{2}$ and $\left|E_{2}(z)\right|^{2}=\left|\check{E}_{2}(z)\right|^{2}$. Finally, the interferences between these signals agree with the measured ones since at $|z|=1,\left|E_{1}(z)+E_{2}(z)\right|^{2}=\mid \check{E}_{1}(z)+$ $\left.\check{E}_{2}(z)\right|^{2}$ and $\left|E_{1}(z)+\mathbf{i} E_{2}(z)\right|^{2}=\left|\check{E}_{1}(z)+\mathbf{i} \check{E}_{2}(z)\right|^{2}$. Thus,

\footnotetext{
${ }^{5}$ For $|z|=1$ and $z_{j}=0$ it holds that $\left(z-z_{j}\right)\left(\bar{z}-\bar{z}_{j}\right)=1$, thus we can ignore these roots.
}

these two signals solve the corresponding vectorial phase retrieval problem too, but are not related to $f_{1}, f_{2}$ by a trivial ambiguity. Hence, the solution is not unique.

\section{APPENDIX II}

\section{PROOF OF THEOREM 2}

Proof of Theorem 2: First note that the true phase vector $\mathrm{x}$ is a minimizer of $Q_{\tau}$ since $\mathrm{x}^{H} Q_{\tau} \mathrm{x}=0$. Let us assume that there exist another minimizer, $\mathrm{y}=\left(y_{1}(\omega), y_{2}(\omega)\right) \in \mathbb{C}^{2 n}$. Since $\mathrm{y}$ is not necessarily a phase vector, we decompose it into amplitude and phase, $y_{1,2}(\omega)=\left|y_{1,2}(\omega)\right| e^{\mathbf{i} \psi_{1,2}(\omega)}$. Being a minimizer, ${ }^{H} Q_{\tau} \mathrm{y}=0$ as well. Thus, $\mathcal{A}_{\tau} \mathrm{y}=0$. The $n$ interference equations imply that $\left|y_{1}(\omega)\right|=\left|y_{2}(\omega)\right|=Y(\omega)$ and also $e^{\mathbf{i}\left(\psi_{1}(\omega)-\phi_{1}(\omega)\right)}=e^{\mathbf{i}\left(\psi_{2}(\omega)-\phi_{2}(\omega)\right)}$.

Let $H_{1}(\omega)$ and $H_{2}(\omega)$ be defined as follows,

$$
\begin{aligned}
H_{1}(\omega) & =\left|F_{1}(\omega)\right| y_{1}(\omega) \\
& =F_{1}(\omega) Y(\omega) e^{\mathbf{i}\left(\psi_{1}(\omega)-\phi_{1}(\omega)\right)} \\
H_{2}(\omega) & =\left|F_{2}(\omega)\right| y_{2}(\omega) \\
& =F_{2}(\omega) Y(\omega) e^{\mathbf{i}\left(\psi_{2}(\omega)-\phi_{2}(\omega)\right)} .
\end{aligned}
$$

The $2(n-\tau)$ equations for vanishing residuals $R_{1}$ and $R_{2}$ imply that $H_{1}$ and $H_{2}$ are the Fourier Transforms of two signals $h_{1}$, $h_{2}$ with a support of length $\tau$. Hence, they may be decomposed as follows,

$$
H_{1}(z)=C_{3} \prod_{j=1}^{s}\left(z-z_{j}^{H 1}\right) H_{2}(z)=C_{4} \prod_{j=1}^{s}\left(z-z_{j}^{H 2}\right) .
$$

Similarly, the FT of $f_{1}, f_{2}$ can also be factored as

$$
F_{1}(z)=C_{1} \prod_{j=1}^{s}\left(z-z_{j}^{F 1}\right) F_{2}(z)=C_{2} \prod_{j=1}^{s}\left(z-z_{j}^{F 2}\right)
$$

where as in the proof of Thm. 1, $z=e^{\mathbf{i} \omega}$. Using $Y(z) e^{\mathrm{i}\left(\psi_{1}(z)-\phi_{1}(z)\right)}=\frac{H_{1}(z)}{F_{1}(z)}=\frac{H_{2}(z)}{F_{2}(z)}$ we get

$$
\frac{C_{3} \prod\left(z-z_{j}^{H 1}\right)}{C_{1} \prod\left(z-z_{j}^{F 1}\right)}=\frac{C_{4} \prod\left(z-z_{j}^{H 2}\right)}{C_{2} \prod\left(z-z_{j}^{F 2}\right)}
$$

or, equivalently

$$
\prod\left(z-z_{j}^{H 2}\right) \prod\left(z-z_{j}^{F 1}\right)=C \prod\left(z-z_{j}^{F 2}\right) \prod\left(z-z_{j}^{H 1}\right)
$$

But by spectral independence $\left\{z_{j}^{F 1}\right\} \cap\left\{z_{j}^{F^{2}}\right\}=\emptyset$. Thus $\left\{z_{j}^{H 1}\right\}=\left\{z_{j}^{F 1}\right\}$ and $\left\{z_{j}^{H^{2}}\right\}=\left\{z_{j}^{F^{2}}\right\}$. Moreover $H_{1}\left(\omega_{j}\right) \propto F_{1}\left(\omega_{j}\right)$ and $H_{2}\left(\omega_{j}\right) \propto F_{2}\left(\omega_{j}\right)$, meaning $Y\left(\omega_{j}\right)=$ Const and $e^{\mathbf{i} \psi_{1}\left(\omega_{j}\right)}=e^{\mathbf{i} \phi_{1}\left(\omega_{j}\right)}$ up to a global phase.

\section{APPENDIX III}

\section{Statistical Properties of the Residuals $R_{1}, R_{2}, R_{3}$}

Low Noise Assumption: In calculating the statistical properties of the different residuals, we assume a high signal to noise ratio, such that in (3), with high probability both $\frac{\eta_{i}^{s}\left(\omega_{j}\right)}{\left|F_{i}\left(\omega_{j}\right)\right|} \ll 1$ and $\frac{\eta_{i}^{s h}}{\left|F\left(\omega_{j}\right)\right|} \ll 1$. The latter condition implies that the shot noise, 
which typically follows a Poisson distribution, may be approximated by a $\mathcal{N}(0,1)$ r.v.. We note that if at some frequency the Fourier coefficient is comparable to the noise level or is even identically zero, these conditions do not hold there, and the developments below are not valid. However, as discussed in the paper, the reconstruction of the signal is quite insensitive to the exact value of estimated phases at such frequencies. Under these assumptions and suppressing the dependence on $\omega_{j}$, we can approximate the spectral amplitudes as follows,

$$
\begin{aligned}
\left|\tilde{F}_{i}\right| & =\sqrt{\left|F_{i}+\eta_{i}^{s}\right|^{2}+\left|F_{i}+\eta_{i}^{s}\right| \eta_{i}^{s h}} \\
& \approx\left|F_{i}+\eta_{i}^{s}\right|\left(1+\frac{\eta_{i}^{s h}}{2\left|F_{i}+\eta_{i}^{s}\right|}\right) \\
& \approx\left|F_{i}\right|\left(1+\frac{\Re\left\{\eta_{i}^{s} e^{-\mathbf{i} \phi_{i}\left(\omega_{j}\right)}\right\}}{\left|F_{i}\left(\omega_{j}\right)\right|}\right)\left(1+\frac{\eta_{i}^{s h}}{2\left|F_{i}+\eta_{i}^{s}\right|}\right) \\
& \approx\left|F_{i}\right|\left(1+\frac{\Re\left\{\eta_{i}^{s} e^{-\mathbf{i} \phi_{i}\left(\omega_{j}\right)}\right\}}{\left|F_{i}\right|}+\frac{\eta_{i}^{s h}}{2\left|F_{i}\right|}\right) .
\end{aligned}
$$

In other words, at the high SNR regime, we may approximate

$$
\left|\tilde{F}_{i}\left(\omega_{j}\right)\right| \simeq\left|F_{i}\left(\omega_{j}\right)\right|+\eta_{i}\left(\omega_{j}\right) \quad \text { for } i=1 \ldots 4
$$

where

$$
\eta_{i}\left(\omega_{j}\right)=\Re\left\{\eta_{i}^{s}\left(\omega_{j}\right) e^{-\mathbf{i} \phi_{i}\left(\omega_{j}\right)}\right\}+\frac{1}{2} \eta_{i}^{s h}\left(\omega_{j}\right) .
$$

Note that if both $\eta_{i}^{s h}$ and $\eta_{i}^{s}$ are zero mean Gaussian then $\eta_{i}\left(\omega_{j}\right)$ is zero mean Gaussian as well. However, it is not independent of the signal, due to the factor $e^{-\mathbf{i} \phi_{i}\left(\omega_{j}\right)}$ multiplying the first term in (35) above.

For future use, we first state and prove the following auxiliary lemma regarding the distribution of $\eta_{i}\left(\omega_{j}\right)$.

Lemma 1: Let $\eta_{i}\left(\omega_{j}\right)$ be given by (35). Then, $\eta_{i}\left(\omega_{j}\right)$ is a Gaussian random variable with zero mean, and variance

$$
\mathbb{E}\left[\eta_{i}\left(\omega_{j}\right)^{2}\right]=\frac{1}{4}+\frac{\sigma^{2}}{2} .
$$

In addition, $\mathbb{E}\left[\eta_{i}\left(\omega_{j}\right) \eta_{\ell}\left(\omega_{k}\right)\right]=0$ for $i \neq \ell$ or $j \neq k$.

Proof: Using (35) and the assumption that the shot noise and signal noise are uncorrelated, we have

$$
\mathbb{E}\left[\eta_{i}\left(\omega_{j}\right)^{2}\right]=\frac{1}{4} \mathbb{E}\left[\eta_{i}^{s h}\left(\omega_{j}\right)^{2}\right]+\mathbb{E}\left[\Re\left\{\eta_{i}^{s}\left(\omega_{j}\right) e^{-\mathbf{i} \phi_{i}\left(\omega_{j}\right)}\right\}^{2}\right] .
$$

The assumption $\eta_{i}^{s h}\left(\omega_{j}\right) \sim \mathcal{N}(0,1)$ gives $\mathbb{E}\left[\left|\eta_{i}^{s h}\left(\omega_{j}\right)\right|^{2}\right]=1$.

As for the second term, since $\eta_{i}^{s}\left(\omega_{j}\right) \sim \mathbb{C N}\left(0, \sigma^{2}\right)$ we may write $\eta_{i}^{s}\left(\omega_{j}\right)=\sigma \frac{a_{j}+\mathbf{i} b_{j}}{\sqrt{2}}$ where $a_{j}$ and $b_{j}$ are i.i.d. $\mathcal{N}(0,1)$. Thus, $\Re\left\{\eta_{i}^{s}\left(\omega_{j}\right) e^{-\mathbf{i} \phi_{i}\left(\omega_{j}\right)}\right\}=\frac{\sigma}{\sqrt{2}}\left(a_{j} \cos \phi_{j}+b_{j} \sin \phi_{j}\right)$ and its variance is $\sigma^{2} / 2$. Hence, (36) follows.

The fact that $\mathbb{E}\left[\eta_{i}\left(\omega_{j}\right) \eta_{\ell}\left(\omega_{k}\right)\right]=0$ for $i \neq \ell$ or $j \neq k$ follows from our assumption that noise is uncorrelated between different frequencies and across different experiments.

Statistical Properties of the Residuals $R_{1}$ and $R_{2}$ : Let us first study the distribution of the residual $R_{1}\left(t_{k}\right)$ of (4), when evaluated at the true unknown phases, for $t_{k}>\tau$. Combining
(3) and (4) with (34) and the assumption of a finite support gives, up to second order noise terms $\mathcal{O}\left(\mathbb{E}\left[\eta_{1}^{2}\right]\right)$,

$$
R_{1}\left(t_{k}\right)=\frac{1}{n} \sum_{j} \eta_{1}\left(\omega_{j}\right) x_{1}\left(\omega_{j}\right) e^{\mathrm{i} \omega_{j} t_{k}}
$$

That is, at the true phases, the residual values $R_{1}\left(t_{k}\right)$ depend both on the noise realization and on the unknown phase vector $x_{1}$. The following lemma characterizes some statistical properties of this residual. Analogous results hold for the second residual $R_{2}$.

Lemma 2: In the limit of high SNR, the random vector of residuals $R_{1}\left(t_{k}\right), t_{k}>\tau$ evaluated at the exact (unknown) phase vector $x_{1}$ satisfies the following properties:

1) $\mathbb{E}\left[R_{1}\left(t_{k}\right)\right]=0$.

2) $\mathbb{E}\left[R_{1}\left(t_{k}\right) \overline{R_{1}\left(t_{m}\right)}\right]=\frac{1}{n}\left(\frac{1}{4}+\frac{\sigma^{2}}{2}\right) \delta_{k m}$

3) $\mathbb{E}\left[R_{1}\left(t_{k}\right) R_{1}\left(t_{m}\right)\right]$

$\left(\frac{1}{4}+\frac{\sigma^{2}}{2}\right) \frac{1}{n^{2}} \sum_{j=1}^{n} x_{1 j}^{2} e^{\mathbf{i} \omega_{j}\left(t_{k}+t_{m}\right)}$

Note that since the sum in property (3) is in general not zero, the residuals $R_{1}\left(t_{k}\right)$ and $R_{1}\left(t_{m}\right)$ are unfortunately not independent, and moreover their correlation depends on the unknown phase vector $x_{1}$. However, for a random phase vector with $x_{1 j}=e^{\mathbf{i} \theta_{j}}$ where $\theta_{j}$ are all i.i.d. uniform on $[0,2 \pi]$, this (random) sum is $\mathcal{O}(\sqrt{n})$. Hence, for $n \gg 1$, with high probability, the covariance between the two residuals of $\mathcal{O}\left(n^{-1.5}\right)$, is negligible compared to $\mathbb{E}\left[R_{1}\left(t_{k}\right) \overline{R_{1}\left(t_{k}\right)}\right]=\mathcal{O}\left(n^{-1}\right)$.

Neglecting these correlations yields (5).

Proof: Property (1) follows directly from the linearity of the expectation operator,

$$
\mathbb{E}\left[R_{1}\left(t_{k}\right)\right]=\frac{1}{n} \sum_{j} \mathbb{E}\left[\eta_{1}\left(\omega_{j}\right)\right] x_{1}\left(\omega_{j}\right) e^{\mathrm{i} \omega_{j} t_{k}}=0 .
$$

Next, using Lemma 1 and the fact that $\left|x_{1}\left(\omega_{j}\right)\right|=1$ gives

$$
\begin{aligned}
\mathbb{E}\left[R_{1}\left(t_{k}\right) \overline{R_{1}\left(t_{m}\right)}\right] & =\mathbb{E}\left[\frac{1}{n^{2}} \sum_{j, l} \eta_{1 j} x_{1 j} e^{\mathbf{i} \omega_{j} t_{k}} \eta_{1 l} \overline{x_{1 l}} e^{-\mathbf{i} \omega_{l} t_{m}}\right] \\
& =\frac{1}{n^{2}} \sum_{j} \mathbb{E}\left[\eta_{1 j}^{2}\right] e^{\mathbf{i} \omega_{j}\left(t_{k}-t_{m}\right)}
\end{aligned}
$$

where $\eta_{1 j}=\eta_{1}\left(\omega_{j}\right)$ and $x_{i j}=x_{1}\left(\omega_{j}\right)$. Property (2) readily follows from (36) for $\mathbb{E}\left[\left|\eta_{1}\left(\omega_{j}\right)\right|^{2}\right]$. Similarly, we have that

$$
\begin{aligned}
\mathbb{E}\left[R_{1}\left(t_{k}\right) R_{1}\left(t_{m}\right)\right] & =\mathbb{E}\left[\frac{1}{n^{2}} \sum_{j, l} \eta_{1 j} x_{1 j} e^{\mathrm{i} \omega_{j} t_{k}} \eta_{1 l} x_{1 l} e^{\mathrm{i} \omega_{l} t_{m}}\right] \\
& =\frac{1}{n^{2}} \sum_{j} \mathbb{E}\left[\eta_{1 j}^{2}\right] x_{1 j}^{2} e^{\mathrm{i} \omega_{j}\left(t_{k}+t_{m}\right)}
\end{aligned}
$$

Using (36) yields property (3).

The Interference Residual $R_{3}$ : The following lemma describes some statistical properties of the interference residuals $R_{3}\left(\omega_{j}\right)$.

Lemma 3: The random vector of residuals $R_{3}\left(\omega_{j}\right)$, evaluated at the phase vectors $x_{1}, x_{2}$ has the following properties: 
1) Up to second order terms in the noises $\eta_{1 \ldots 4}, R_{3}\left(\omega_{j}\right)$ can be written as a linear combination of $\eta_{i}$ as follows,

$$
R_{3}\left(\omega_{j}\right)=x_{2}\left(\omega_{j}\right) \sum_{h=1}^{4} A_{h}\left(\omega_{j}\right) \eta_{h}\left(\omega_{j}\right)+\mathcal{O}\left(\mathbb{E}\left[\eta\left(\omega_{j}\right)^{2}\right]\right)
$$

where $A_{h}\left(\omega_{j}\right)$ depend on the measured spectral intensities $\left|\tilde{F}_{i}\left(\omega_{j}\right)\right|$.

2) Neglecting the second order terms in (40), $R_{3}\left(\omega_{j}\right)$ are independent zero mean Gaussian random variables, with variance

$$
\mathbb{E}\left[\left|R_{3}\left(\omega_{j}\right)\right|^{2}\right]=\left(\frac{1}{4}+\frac{\sigma^{2}}{2}\right) \sum_{h=1}^{4} A_{h}^{2}\left(\omega_{j}\right)+\mathcal{O}\left(\mathbb{E}\left[\eta\left(\omega_{j}\right)^{4}\right]\right) .
$$

Proof: To prove property (1), we expand $\tilde{G}\left(\omega_{j}\right)$ to first order in the additive noises. Combining (10), (12) and (35), suppressing the dependence on $\omega_{j}$ and denoting quadratic terms in $\eta_{i}$ by $\mathcal{O}\left(\eta^{2}\right)$ :

$$
\begin{aligned}
\tilde{G}= & G\left(1-\frac{\eta_{1}}{\left|F_{1}\right|}-\frac{\eta_{2}}{\left|F_{2}\right|}\right) \\
& +\frac{\left|F_{3}\right| \eta_{3}+\mathbf{i}\left|F_{4}\right| \eta_{4}-(1+\mathbf{i})\left(\left|F_{1}\right| \eta_{1}+\left|F_{2}\right| \eta_{2}\right)}{\left|\tilde{F}_{1}\right|\left|\tilde{F}_{2}\right|}+\mathcal{O}\left(\mathbb{E}\left[\eta^{2}\right]\right) .
\end{aligned}
$$

Since $\left|\tilde{F}_{i}\right|=\left|F_{i}\right|+\eta_{i}+\mathcal{O}\left(\mathbb{E}\left[\eta_{i}^{2}\right]\right)$, we can replace $G$ by $\tilde{G}$ and each $\left|\tilde{F}_{i}\right|$ by $\left|F_{i}\right|$ in the equation above. Namely,

$$
\tilde{G}\left(\omega_{j}\right)=G\left(\omega_{j}\right)+\sum_{h=1}^{4} b_{h}\left(\omega_{j}\right) \eta_{h}\left(\omega_{j}\right)+\mathcal{O}\left(\mathbb{E}\left[\eta^{2}\right]\right)
$$

with

$$
b_{1}=-\frac{\tilde{G}}{\left|\tilde{F}_{1}\right|}-\frac{1+\mathbf{i}}{\left|\tilde{F}_{2}\right|} \quad b_{2}=-\frac{\tilde{G}}{\left|\tilde{F}_{2}\right|}-\frac{1+\mathbf{i}}{\left|\tilde{F}_{1}\right|}
$$

and

$$
b_{3}=\frac{\left|\tilde{F}_{3}\right|}{\left|\tilde{F}_{1}\right|\left|\tilde{F}_{2}\right|}, \quad b_{4}=\frac{\mathbf{i}\left|\tilde{F}_{4}\right|}{\left|\tilde{F}_{1}\right|\left|\tilde{F}_{2}\right|} .
$$

Next, we consider the residual $R_{3}\left(\omega_{j}\right)$. Combining (42) with (11), and suppressing the dependence on $\omega_{j}$ gives

$$
\begin{aligned}
R_{3} & =x_{1}-x_{2} \frac{G+\sum_{h=1}^{4} b_{h} \eta_{h}}{\left|G+\sum_{h=1}^{4} b_{h} \eta_{h}\right|}+\mathcal{O}\left(\mathbb{E}\left[\eta^{2}\right]\right) \\
& =x_{1}-x_{2} \frac{G+\sum_{h=1}^{4} b_{h} \eta_{h}}{|G|\left(1+\sum_{h=1}^{4} \Re\left(\frac{b_{h}}{G}\right) \eta_{h}\right)}+\mathcal{O}\left(\mathbb{E}\left[\eta^{2}\right]\right) .
\end{aligned}
$$

Since $|G|=1$ and $x_{1}-x_{2} G=0$ we obtain

$$
\begin{aligned}
R_{3} & =-x_{2}\left(\frac{\sum_{h=1}^{4} b_{h} \eta_{h}}{|\tilde{G}|}-\frac{\tilde{G}}{|\tilde{G}|} \sum_{h=1}^{4} \Re\left(\frac{b_{h}}{\tilde{G}}\right) \eta_{h}\right)+\mathcal{O}\left(\mathbb{E}\left[\eta^{2}\right]\right) \\
& =-\frac{x_{2}}{|\tilde{G}|} \sum_{h=1}^{4}\left(b_{h}-\tilde{G} \Re\left(\frac{b_{h}}{\tilde{G}}\right)\right) \eta_{h}+\mathcal{O}\left(\mathbb{E}\left[\eta^{2}\right]\right)
\end{aligned}
$$

Hence, (40) follows with coefficients $A_{h}\left(\omega_{j}\right)$ given by

$$
A_{h}\left(\omega_{j}\right)=\frac{-1}{\left|\tilde{G}\left(\omega_{j}\right)\right|}\left(b_{h}\left(\omega_{j}\right)-\tilde{G}\left(\omega_{j}\right) \Re\left(\frac{b_{h}\left(\omega_{j}\right)}{\tilde{G}\left(\omega_{j}\right)}\right)\right)
$$

and $b_{h}$ given by (43)-(44). This proves property (1).

To prove property (2), note that by Lemma $1, \eta_{i}\left(\omega_{j}\right)$ are all independent $\mathcal{N}\left(0, \frac{1}{4}+\frac{\sigma^{2}}{2}\right)$ random variables. As a linear combination of Gaussian i.i.d. is also Gaussian, property (2) follows. Moreover, as $x_{2}\left(\omega_{j}\right)$ are phases with unit norm and the mean of $\eta^{3}$ is zero, (41) follows as well.

Correlations Between $R_{3}$ and $R_{1,2}$ : The following lemma shows that $R_{3}\left(\omega_{j}\right)$ is not independent of $R_{1}\left(t_{k}\right)$ and $R_{2}\left(t_{k}\right)$, and their covariance depends on the unknown phase vectors $x_{1,2}$. However, it asserts that their correlation is small, of $\mathcal{O}\left(\frac{1}{\sqrt{n}}\right)$.

Lemma 4: Up to second order terms in the noises, $\mathcal{O}\left(\mathbb{E}\left[\eta^{2}\right]\right)$, the covariance between $R_{3}\left(\omega_{j}\right)$ and $R_{1,2}\left(t_{k}\right)$, when evaluated at the true phase vectors $x_{1}, x_{2}$ is given by

$$
\mathbb{E}\left[R_{3}\left(\omega_{j}\right) R_{1}\left(t_{k}\right)\right]=\left(\frac{1}{4}+\frac{\sigma^{2}}{2}\right) A_{1}\left(\omega_{j}\right) x_{2}\left(\omega_{j}\right) x_{1}\left(\omega_{j}\right) e^{\mathrm{i} \omega_{j} t_{k}}
$$

with similar formulas for $\mathbb{E}\left[R_{3}\left(\omega_{j}\right) R_{2}\left(t_{k}\right)\right]$ and for $\mathbb{E}\left[R_{3} \bar{R}_{1}\right]$.

Note that by this lemma and property (2) of Lemma 2 , the correlation between $R_{3}$ and $R_{1}$ or $R_{2}$ is $\mathcal{O}\left(\frac{1}{\sqrt{n}}\right)$.

Proof: Using the independence properties of $\eta_{i}$, up to second order noise terms $\mathcal{O}\left(\mathbb{E}\left[\eta^{2}\right]\right)$,

$$
\begin{aligned}
\mathbb{E}\left[R_{3}\left(\omega_{j}\right) R_{1}\left(t_{k}\right)\right] & =\mathbb{E}\left[x_{2 j} \sum_{h=1}^{4} A_{h}\left(\omega_{j}\right) \eta_{h j} \sum_{l} \eta_{1 l} x_{1 l} e^{\mathrm{i} \omega_{l} t_{k}}\right] \\
& =x_{2 j} A_{1}\left(\omega_{j}\right) \mathbb{E}\left[\eta_{1, j}^{2}\right] x_{1 j} e^{\mathrm{i} \omega_{j} t_{k}} .
\end{aligned}
$$

The lemma now readily follows from (36).

\section{ACKNOWLEDGMENT}

We thank Prof. Yonina Eldar for interesting discussions, and the anonymous referees for many valuable comments and suggestions.

\section{REFERENCES}

[1] D. R. Luke, J. Burke, and R. Lyon, "Optical wavefront reconstruction: Theory and numerical methods," SIAM Rev., vol. 44, pp. 169-224, 2002. 
[2] S. Marchesini, "A unified evaluation of iterative projection algorithms for phase retrieval,” Rev. Sci. Instrum., vol. 78, pp. 011301-011301, 2007.

[3] R. P. Millane, "Phase retrieval in crystallography and optics," J. Opt. Soc. Amer. A, vol. 7, pp. 394-411, 1990.

[4] P. McDonald and A. Lonergana, "Two-dimensional Fourier transform NMR imaging of solids using multiple pulse line narrowing," Physica $B$, vol. 176, no. 3, pp. 173-179, 1992.

[5] J. C. Dainty and J. R. Fienup, Image Recovery-Theory and Application. London: Academic, ch. 7, pp. 1987-1987.

[6] H. N. Chapman et al., "Femtosecond diffractive imaging with a soft-X-ray free-electron laser," Nature Phys., vol. 2, no. 12, pp. 839-843, 2006.

[7] H. Quiney, A. Peele, Z. Cai, D. Paterson, and K. Nugent, "Diffractive imaging of highly focused X-ray fields," Nature Phys., vol. 2, no. 2, pp. 101-104, 2006.

[8] K. J. Gaffney and H. N. Chapman, "Imaging atomic structure and dynamics with ultrafast x-ray scattering," Sci., vol. 316, 2007.

[9] A. Szameit, Y. Shechtman, E. Osherovich, E. Bullkich, P. Sidorenko, H. Dana, S. Steiner, E. B. Kley, S. Gazit, T. Cohen-Hyams, S. Shoham, M. Zibulevsky, I. Yavneh, Y. C. Eldar, O. Cohen, and M. Segev, "Sparsity-based single-shot subwavelength coherent diffractive imaging," Nature Mater., vol. 11, pp. 455-459, 2012.

[10] H. Chapman and K. Nugent, "Coherent lensless X-ray imaging," Nature Photon., vol. 4, pp. 833-839, 2010

[11] H. Jiang, C. Song, C.-C. Chen, R. Xu, K. Raines, B. Fahimian, C.-H. Lu, T.-K. Lee, A. Nakashima, J. Urano, T. Ishikawa, F. Tamanoi, and J. Miao, "Quantitative 3d imaging of whole, unstained cells by using x-ray diffraction microscopy," Proc. Nat. Acad. Sci., vol. 107, no. 25, pp. 11 234-11 239, 2010

[12] P. Jaming, "Phase retrieval techniques for radar ambiguity problems," J. Fourier Anal. Appl., vol. 5, pp. 309-329, 1999.

[13] R. Trebino, K. W. DeLong, D. N. Fittinghoff, J. N. Sweetser, M. A. Krumbugel, and B. A. Richman, "Measuring ultrashort laser pulses in the time-frequency domain using frequency-resolved optical gating," Rev. Sci. Instrum., vol. 68, no. 9, pp. 3277-3295, 1997.

[14] I. A. Walmsley and C. Dorrer, "Characterization of ultrashort electromagnetic pulses," Adv. Opt. Photon., vol. 1, pp. 308-437, 2009.

[15] Y. Bruck and L. Sodin, "On the ambiguity of the image reconstruction problem,” Opt. Commun., vol. 30, no. 3, pp. 304-308, 1979.

[16] R. Gerchberg and W. Saxton, "A practical algorithm for the determination of phase from image and diffraction plane pictures," Optik, vol. 35, pp. 235-246, 1972.

[17] J. R. Fienup, "Phase retrieval algorithms: A comparison," App. Opt., vol. 21 , no. 15 , pp. 2758-2769, 1982.

[18] H. Bauschke, P. Combettes, and D. R. Luke, "Phase retrieval, error reduction algorithm, and Fienup variants: A view from convex optimization," J. Opt. Soc. Amer. A., vol. 19, pp. 1334-45, 2002.

[19] J. N. Cederquist and C. C. Wackerman, "Phase-retrieval error: A lower bound," J. Opt. Soc. Am. A, vol. 4, pp. 1788-1792, 1987.

[20] J. Sanz, "Mathematical considerations for the problem of Fourier transform phase retrieval from magnitude," SIAM J. Appl. Math., vol. 45, pp. 651-664, 1985.

[21] M. Klibanov, P. E. Sacks, and A. V. Tikhonravov, "The phase retrieval problem," Inverse Problems, vol. 11, pp. 1-28, 1995.

[22] O. Raz, O. Schwartz, D. Austin, A. S. Wyatt, A. Schiavi, O. Smirnova, B. Nadler, I. A. Walmsley, D. Oron, and N. Dudovich, "Vectorial phase retrieval for linear characterization of attosecond pulses," Phys. Rev. Lett., vol. 107, p. 133902, 2011.

[23] D. R. Austin, T. Witting, C. A. Arrell, F. Frank, A. S. Wyatt, J. P. Marangos, J. W. Tisch, and I. A. Walmsley, "Lateral shearing interferometry of high-harmonic wavefronts," Opt. Lett., vol. 36, no. 10, pp. 1746-1748, 2011.

[24] J. Walker, "The phase retrieval problem, a solution based on zero location by exponential apodization," Optica Acta, vol. 28, pp. 735-738, 1981.

[25] J. W. Wood, M. A. Fiddy, and R. E. Burge, "Phase retrieval using two intensity measurements in the complex plane," Opt. Lett., vol. 6, pp. 514-516, 1981.

[26] L. J. Allen and M. P. Oxley, "Phase retrieval from series of images obtained by defocus variation," Opt. Commun., vol. 199, pp. 65-75, 2001.

[27] T. E. Gureyev, A. Roberts, and K. A. Nugent, "Phase retrieval with the transport-of-intensity equation: Matrix solution with use of zernike polynomials," J. Opt. Soc. Amer. A, vol. 12, pp. 1932-1941, 1995.
[28] E. Osherovich, M. Zibulevsky, and I. Yavneh, Phase Retrieval Combined With Digital Holography 2012 [Online]. Available: http://arxiv. org/pdf/1203.0853v1.pdf

[29] E. J. Candes, Y. Eldar, T. Strohmer, and V. Voroninski, "Phase retrieval via matrix completion," SIAM J. Imag. Sci., 2012.

[30] H. Faulkner and J. M. Rodenburg, "Movable aperture lensless transmission microscopy: A novel phase retrieval algorithm," Phys. Rev. Lett., vol. 93, no. 2, p. 023903, 2004.

[31] H. Ohlsson, A. Y. Yang, R. Dong, and S. Sastry, "Compressive phase retrieval from squared output measurements via semidefinite programming," in Proc. 16th IFAC Symp. Syst. Identif., 2012.

[32] E. J. Candes, T. Strohmer, and V. Voroninski, "Phaselift: Exact and stable signal recovery from magnitude measurements via convex programming," Commun. Pure Appl. Math., 2012.

[33] L. Demanet and P. Hand, "Stable optimizationless recovery from phaseless linear measurements," 2012 [Online]. Available: http://arxiv.org/abs/1208.1803

[34] I. Waldspurger, A. D'Aspremont, and S. Mallat, "Phase recovery, maxcut and complex semidefinite programming," 2012 [Online]. Available: http://arxiv.org/abs/1206.0102

[35] R. Balan, B. Bodmann, P. Casazza, and D. Edidin, "Painless reconstruction from magnitudes of frame coefficients," J. Fourier Anal. Appl., vol. 15, pp. 488-501, 2009.

[36] D. W. Kammler, A First Course in Fourier Analysis. : Cambridge University press, 2008.

[37] A. Yagle, "1-d and 2-d minimum and non-minimum phase retrieval by solving linear systems of equations," in IEEE Int. Conf. Acoust., Speech, Signal Process., 1996.

[38] G. Thakur, "Reconstruction of bandlimited functions from unsigned samples," J. Fourier Anal. Appl, vol. 17, pp. 720-732, 2011.

[39] M. Chu and J. Watterson, "On a multivariate eigenvalue problem: I. Algebraic theory and power method," SIAM J. Sci. Comput., vol. 14, pp. 1089-1106, 1993.

[40] L.-H. Zhang, "Riemannian newton method for the multivariate eigenvalue problem," SIAM J. Matrix Anal. Appl., vol. 31, no. 5, pp. 2972-2996, 2010.

[41] L.-H. Zhang and L.-Z. Liao, "An alternating variable method for the maximal correlation problem," J. Glob. Optim., vol. 54, pp. 199-218, 2012 .

[42] A. Singer, "Angular synchronization by eigenvectors and semidefinite programming," Appl. Computat. Harmon. Anal., vol. 30, pp. 20-36, 2011 .

Oren Raz received the B.Sc. degree in mathematics and physics (cum laude) from the Hebrew University of Jerusalem, Israel, and the M.Sc. degree in physics from the Technion, Israel.

He is currently a Ph.D. student with the faculty of Physics, Weizmann Institute of Science, Israel.

Nirit Dudovich received the B.Sc. degree from Tel Aviv University, Israel, and the M.Sc. and Ph.D. degrees from the Weizmann Institute of Science, Israel, in the field of quantum coherent control.

After a Postdoctoral Research term with the National Research Council, Ottawa, Canada, working in the field of attosecond science, she joined the faculty of Physics, Weizmann Institute of Science, Israel, as a Senior Scientist in 2007. Her research is concerned with basic phenomena in strong field light-matter interactions, focusing in particular on the generation and measurement of attosecond processes.

Boaz Nadler (M'10) received the Ph.D. degree in applied mathematics from Tel Aviv University, Israel.

From 2002 to 2005, he was a Gibbs Instructor/Assistant Professor with the Department of Mathematics, Yale University, New Haven, CT. He is currently an Associate Professor in the Department of Computer Science and Applied Mathematics, Weizmann Institute of Science, Israel. His research interests are in mathematical statistics, statistical machine learning and their applications in chemometrics, and in signal and image processing. 